\title{
Genetic characterization of an almond germplasm collection and volatilome profiling of raw and roasted kernels
}

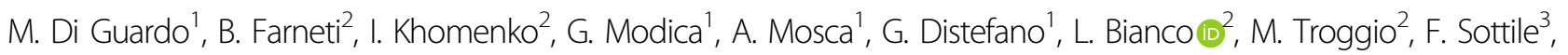
S. La Malfa', F. Biasioli ${ }^{2}$ and A. Gentile ${ }^{1,4}$

\begin{abstract}
Almond is appreciated for its nutraceutical value and for the aromatic profile of the kernels. In this work, an almond collection composed of 96 Sicilian accessions complemented with 10 widely cultivated cultivars was phenotyped for the production of volatile organic compounds using a proton-transfer time-of-flight mass spectrometer and genotyped using the Illumina Infinium $18 \mathrm{~K}$ Peach SNP array. The profiling of the aroma was carried out on fresh and roasted kernels enabling the detection of 150 mass peaks. Sixty eight, for the most related with sulfur compounds, furan containing compounds, and aldehydes formed by Strecker degradation, significantly increased during roasting, while the concentration of fifty-four mass peaks, for the most belonging to alcohols and terpenes, significantly decreased. Four hundred and seventy-one robust SNPs were selected and employed for population genetic studies. Structure analysis detected three subpopulations with the Sicilian accessions characterized by a different genetic stratification compared to those collected in Apulia (South Italy) and the International cultivars. The linkagedisequilibrium (LD) decay across the genome was equal to $r^{2}=0.083$. Furthermore, a high level of collinearity $\left(r^{2}=\right.$ 0.96) between almond and peach was registered confirming the high synteny between the two genomes. A preliminary application of a genome-wide association analysis allowed the detection of significant marker-trait associations for 31 fresh and 33 roasted almond mass peaks respectively. An accurate genetic and phenotypic characterization of novel germplasm can represent a valuable tool for the set-up of marker-assisted selection of novel cultivars with an enhanced aromatic profile.
\end{abstract}

\section{Introduction}

Almond (Prunus dulcis Mill. D.A. Webb; syn. Prunus amygdalus Batsch.; Amygdalus communis L.; Amygdalus dulcis Mill.), belongs to the genus Prunus, family Rosaceae a taxonomic group that includes numerous species of agronomical interest such as: apple, pear, peach, apricot, cherry, prune, and several berry fruits. Among tree nuts, almond ranks third in worldwide production behind cashew and

\footnotetext{
Correspondence: G Distefano (distefag@unict.it)

${ }^{1}$ Department of Agriculture, Food and Environment (Di3A), University of

Catania, via Valdisavoia 5, 95123 Catania, Italy

${ }^{2}$ Research and Innovation Centre, Fondazione Edmund Mach, San Michele all' Adige, Trento, Italy

Full list of author information is available at the end of the article

These authors contributed equally: Di Guardo M, Farneti B
}

walnut, with the US being the largest producer ${ }^{1}$. In ancient times, its cultivation rapidly spread throughout the Mediterranean regions from central Asia reaching Sicily during the Greek domination ${ }^{2}$. Nowadays, almond is widely cultivated all over the Mediterranean basin.

Almond cultivation relies mainly on a few highly productive cultivars. However, almond germplasm is composed by thousands of accessions showing wide variability in terms of adaptation to different pedoclimatic conditions, resistance to biotic and abiotic stress and kernel quality traits ${ }^{3-5}$. The self-incompatibility of most of the almond cultivars, together with the extensive use of seeds for propagation, played an important role in the differentiation of such massive genetic diversity within the almond species ${ }^{6,7}$.

\section{(c) The Author(s) 2021}

(c) (i) Open Access This article is licensed under a Creative Commons Attribution 4.0 International License, which permits use, sharing, adaptation, distribution and reproduction cc) in any medium or format, as long as you give appropriate credit to the original author(s) and the source, provide a link to the Creative Commons license, and indicate if changes were made. The images or other third party material in this article are included in the article's Creative Commons license, unless indicated otherwise in a credit line to the material. If material is not included in the article's Creative Commons license and your intended use is not permitted by statutory regulation or exceeds the permitted use, you will need to obtain permission directly from the copyright holder. To view a copy of this license, visit http://creativecommons.org/licenses/by/4.0/. 
One of the leading aspects guiding the choice of the almond cultivar is the kernel quality. Such multi-factorial trait encompasses the physical appearance (colour, texture, size), its nutritional properties and the flavour (aroma and taste) ${ }^{3}$. Almonds are particularly valued for their sensory, nutritional, and health attributes ${ }^{4}$ and kernels are often consumed fresh or as ingredients in processed foods ${ }^{5}$. Considering their wide use for fresh consumption or for confectionery, the flavour of both raw and roasted almond kernels greatly influences their economic value. While taste is determined primarily by nonvolatile metabolites (sugars, organic acids, amino acids) and it is perceived in the mouth, aroma is the result of the interplay of a large array of volatile organic compounds (VOCs) and it is perceived largely by the olfactory receptors. In light of this, aroma profiles of raw and roasted almond have been dissected through several approaches at harvest and during storage. VOC profile of raw almonds is composed for the most by aldehydes such as hexanal, nonanal and benzaldehyde ${ }^{6-9}$, although several ketones, alcohols, alkanes and heterocyclic compounds have been reported ${ }^{10}$. Pyrazines, pyrroles, furans and aldehydes comprised the main volatile compound classes in roasted almonds ${ }^{10}$. The chemical reactions behind the formation of the majority of VOCs in roasted almonds are the Maillard reactions ${ }^{11}$ which produce branched-chain aldehydes, alcohols, sulfur-containing and heterocyclic compounds, while straight chain volatiles reflect heat-induced oxidation during roasting ${ }^{8}$. Several sulfur-containing aroma compounds are de novo produced during roasting by the degradation of sulfurcontaining amino-acids, such as dimethyltrisulfide and 2furfurylthiol, respectively formed by methionine and cysteine $^{9,12}$.

Since aroma involves the perception of a plethora of VOCs, their assessment is crucial to guarantee the selection and marketability of high-quality fruit. Thus, high priority should be given to replace poor flavour cultivars with favourable ones, exploiting the variability already available in nature. However, the analysis of the aroma trait in many samples, necessary to overcome the significant biological and genetic variability among samples, may be laborious and time consuming. VOC phenotyping is currently a limiting step in breeding programs, due to high costs and complex analytical techniques. Another limitation also raised by the elevated, and difficult to be controlled, the interaction between fruit genetics, environmental effects, and product transformation. Even though different cultivars are often characterized by substantial variations in flavour ${ }^{8}$, most plant breeding programs have historically neglected this trait, given its intrinsic complexity and costs to phenotype ${ }^{9,10}$. To correct this inconsistency and incorporate flavour into breeding program routines, it is necessary to identify the sources of flavour variability, understand their genetic architecture, and define cost-effective methods of selection.

According to recent publications, direct injection mass spectrometry (DI-MS) techniques, like Proton Transfer Reaction -Time of Flight- Mass Spectrometry (PTR-ToFMS), are powerful high-throughput phenotyping tool for both genetic and quality-related studies ${ }^{11,12}$. The rapidity and the moderate cost of DI-MS analysis may allow to perform a detailed aroma characterization with a peculiar attention to the VOC fold changes caused by ad hoc storage and transformation experiments. Indeed, this technique has been already applied for the VOC characterization of transformed products, such as fermented $\operatorname{cocoa}^{13}$ and coffee beans ${ }^{14}$, and for genetic association studies of different fruit species ${ }^{12,15,16}$.

The production of these VOCs is controlled by two classes of genes: those encoding enzymes responsible for the synthesis of the end products and those encoding factors regulating the biochemical pathways ${ }^{9}$.

A significant increase in the use of both highthroughput DNA-derived data and advanced phenotyping approaches to dissect the causative genes underlying traits of agronomical interest through marker-trait association approaches ${ }^{17-19}$ has happened in the last decades. To this extent several segregating populations were developed to build the first genetic maps of almond ${ }^{20-22}$. Such studies laid the foundations for QTL analysis approaches to detect genomic regions linked to phytosterol content ${ }^{21,23}$ and other traits related to the physical traits of almond nut and kernel ${ }^{22}$. However, none of these genetic association studies was focused on understanding the genetic aroma regulation of almond kernels.

The high genetic similarity between peach and almond $^{19}$ allowed the development of interspecific almond $\mathrm{x}$ peach segregating population and their use for QTL analyses for traits of agronomical interest such as chilling and heat requirement ${ }^{24}$, brown rot resistance ${ }^{25}$ and 'stone-adhesion/flesh-texture ${ }^{26}$. The availability of high-throughput genotyping platforms enabled the use of genome-wide association study (GWAS) approaches on germplasm collections composed by unrelated individuals ${ }^{27}$. GWAS approaches proved its efficiency in almond ${ }^{28}$ as well as in many other outcrossing tree crops, since they are capable of assessing higher allelic variability and smaller linkage blocks compared to other methods.

In light of this, the set-up of an ex situ germplasm collection is a strategic step both for conservation and breeding purposes. The present work is based on the analysis of an ex situ germplasm collection that was already characterized both phenotypically and genetically highlighting a variability both within Sicilian accessions and between those and the Italian and international elite cultivars $^{29,30}$. Overall, such almond germplasm collection 
Table 1 Volatile organic compounds detected by proton transfer reaction time of flight mass spectrometer (PTR-ToFMS) on fresh and roasted almond kernels, over 106 Prunus dulcis accessions

\begin{tabular}{|c|c|c|c|c|c|c|c|c|c|c|c|c|}
\hline \multirow[t]{2}{*}{$m / z$} & \multirow[t]{2}{*}{ Formula } & \multirow[t]{2}{*}{ Tentative identification } & \multicolumn{4}{|l|}{ Raw } & \multicolumn{4}{|c|}{ Roasted } & \multirow[t]{2}{*}{$P$ value } & \multirow[t]{2}{*}{ Variation } \\
\hline & & & mean & std & $\min$ & $\max$ & mean & std & $\min$ & $\max$ & & \\
\hline 26.016 & $\mathrm{C} 2 \mathrm{H} 2+$ & Common fragment & 6.6 & 4.3 & 1.4 & 32.3 & 7.2 & 5.0 & 1.5 & 34.7 & * & $\nearrow$ \\
\hline 28.019 & $\mathrm{C} 2 \mathrm{H} 4+$ & Common fragment & 3.8 & 19.5 & 0.3 & 229.2 & 0.0 & 0.0 & 0.0 & 0.0 & $* * *$ & $\bigcirc$ \\
\hline 31.018 & $\mathrm{CH} 2 \mathrm{OH}+$ & & 33.7 & 17.5 & 4.0 & 210.0 & 112.5 & 39.0 & 24.0 & 345.9 & $* * *$ & $\nearrow$ \\
\hline 33.033 & $\mathrm{CH} 4 \mathrm{OH}+$ & Methanol & 1626.1 & 841.5 & 91.0 & 8627.8 & 5057.5 & 1832.2 & 842.9 & 15728.8 & $* * *$ & $\bigcirc$ \\
\hline 34.996 & $\mathrm{H} 2 \mathrm{SH}+$ & Hydrogen sulfide & 0.0 & 0.0 & 0.0 & 0.0 & 0.1 & 1.1 & 0.0 & 21.4 & * & $\nearrow$ \\
\hline 39.023 & $\mathrm{C} 3 \mathrm{H} 3+$ & Common fragment & 118.3 & 72.8 & 33.9 & 810.5 & 118.3 & 108.2 & 30.2 & 1322.3 & NS & - \\
\hline 41.039 & $\mathrm{C} 3 \mathrm{H} 5+$ & Common fragment & 60.4 & 34.3 & 17.4 & 334.0 & 45.5 & 37.8 & 11.0 & 408.7 & $* * *$ & ○ \\
\hline 42.012 & & & 2.4 & 1.6 & 0.0 & 13.7 & 4.1 & 2.3 & 0.0 & 18.1 & $* * *$ & $\nearrow$ \\
\hline 42.022 & & & 12.3 & 7.5 & 1.4 & 107.3 & 35.5 & 12.0 & 8.0 & 112.9 & $* * *$ & $\nearrow$ \\
\hline 43.018 & $\mathrm{C} 2 \mathrm{H} 3 \mathrm{O}+$ & Common fragment & 220.6 & 111.8 & 42.6 & 758.1 & 257.9 & 195.6 & 40.5 & 2232.2 & $* *$ & $\nearrow$ \\
\hline 43.030 & $\mathrm{CH}_{2} \mathrm{~N} 2 \mathrm{H}+$ & Cyanamide & 32.5 & 15.7 & 4.8 & 162.7 & 87.0 & 29.6 & 0.0 & 231.1 & $* * *$ & $\nearrow$ \\
\hline 43.055 & $\mathrm{C} 3 \mathrm{H} 7+$ & Common fragment & 36.8 & 18.4 & 13.2 & 177.8 & 21.2 & 15.0 & 5.4 & 103.4 & $* * *$ & ○ \\
\hline 44.025 & & & 9.5 & 4.5 & 2.9 & 31.8 & 9.0 & 5.2 & 1.7 & 44.9 & NS & - \\
\hline 45.033 & $\mathrm{C} 2 \mathrm{H} 4 \mathrm{OH}+$ & Acetaldehyde & 912.5 & 1012.1 & 121.8 & 9916.9 & 1066.6 & 954.7 & 152.2 & 9763.6 & * & $\nearrow$ \\
\hline 47.049 & $\mathrm{C} 2 \mathrm{H} 6 \mathrm{OH}+$ & Ethanol & 457.8 & 453.9 & 23.0 & 3352.0 & 316.7 & 362.3 & 16.2 & 2623.1 & $* * *$ & ○ \\
\hline 49.011 & $\mathrm{CH} 4 \mathrm{SH}+$ & Methanethiol & 0.3 & 0.4 & 0.1 & 6.4 & 18.1 & 16.4 & 0.7 & 141.0 & $* * *$ & $\nearrow$ \\
\hline 53.004 & & & 2.3 & 1.3 & 0.6 & 14.6 & 2.6 & 2.0 & 0.6 & 19.2 & * & $\nearrow$ \\
\hline 53.040 & $\mathrm{C} 4 \mathrm{H} 5+$ & Common fragment & 1.4 & 2.8 & 0.3 & 36.6 & 3.3 & 4.3 & 0.4 & 41.6 & $* * *$ & 0 \\
\hline 53.048 & & & 1.9 & 1.1 & 0.0 & 10.3 & 4.1 & 2.1 & 0.0 & 13.9 & $* * *$ & $\nearrow$ \\
\hline 55.054 & $\mathrm{C} 4 \mathrm{H} 7+$ & Butanal, common fragment & 13.2 & 14.7 & 3.9 & 210.9 & 20.3 & 34.4 & 0.4 & 354.4 & $* * *$ & $\nearrow$ \\
\hline 56.026 & & & 0.5 & 0.3 & 0.1 & 2.6 & 0.0 & 0.0 & 0.0 & 0.0 & $* * *$ & ○ \\
\hline 57.035 & $\mathrm{C} 3 \mathrm{H} 4 \mathrm{OH}+$ & Common fragment & 14.9 & 7.4 & 4.4 & 68.4 & 8.8 & 6.5 & 2.2 & 46.9 & $* * *$ & ○ \\
\hline 57.043 & $\mathrm{C} 2 \mathrm{H} 4 \mathrm{~N} 2 \mathrm{H}+$ & Aminoacetonitrile & 8.2 & 3.0 & 1.0 & 16.7 & 4.9 & 2.4 & 0.0 & 15.0 & $* * *$ & ○ \\
\hline 57.070 & $\mathrm{C} 4 \mathrm{H} 9+$ & 1-Butanol & 47.9 & 38.9 & 10.4 & 492.2 & 19.8 & 17.6 & 5.7 & 155.7 & $* * *$ & ○ \\
\hline 59.049 & $\mathrm{C} 3 \mathrm{H} 6 \mathrm{OH}+$ & Acetone & 114.2 & 147.2 & 19.7 & 1455.2 & 289.7 & 279.1 & 48.0 & 1746.2 & $* * *$ & $\nearrow$ \\
\hline 61.028 & $\mathrm{C} 2 \mathrm{H} 4 \mathrm{O} 2 \mathrm{H}+$ & Acetic acid, fragment of esters & 219.3 & 110.3 & 48.6 & 814.8 & 276.7 & 186.4 & 43.6 & 1297.5 & $* * *$ & $\nearrow$ \\
\hline 61.055 & $\mathrm{C} 3 \mathrm{H} 8 \mathrm{OH}+$ & & 3.2 & 2.2 & 1.1 & 35.8 & 6.6 & 13.9 & 1.0 & 172.2 & $* * *$ & $\nearrow$ \\
\hline 63.012 & $\mathrm{CO} 2 \mathrm{H} 3 \mathrm{O}+$ & Water cluster of carbon dioxide & 0.7 & 0.5 & 0.0 & 6.3 & 0.8 & 0.4 & 0.0 & 3.6 & $* * *$ & $\nearrow$ \\
\hline 63.029 & $\mathrm{C} 2 \mathrm{H} 6 \mathrm{SH}+$ & Dimethyl sulfide & 2.2 & 4.3 & 0.0 & 71.4 & 15.4 & 12.0 & 0.0 & 145.5 & $* * *$ & $\nearrow$ \\
\hline 63.043 & $\mathrm{C} 2 \mathrm{H} 6 \mathrm{O} 2 \mathrm{H}+$ & Water cluster of acetaldehyde & 116.0 & 177.3 & 3.6 & 1634.1 & 81.7 & 105.2 & 1.7 & 1036.0 & ** & $\nearrow$ \\
\hline 65.944 & & & 0.0 & 0.0 & 0.0 & 0.2 & 0.0 & 0.0 & 0.0 & 0.2 & $* * *$ & ○ \\
\hline 67.032 & $\mathrm{C} 3 \mathrm{H} 2 \mathrm{~N} 2 \mathrm{H}+$ & Propanedinitrile & 0.1 & 0.0 & 0.0 & 0.7 & 0.1 & 0.1 & 0.0 & 0.6 & $* * *$ & $\nearrow$ \\
\hline 67.057 & $\mathrm{C} 5 \mathrm{H} 7+$ & Pentanal, common fragment & 1.6 & 1.2 & 0.4 & 7.4 & 2.5 & 1.9 & 0.6 & 20.4 & $* * *$ & $\nearrow$ \\
\hline 67.992 & & & 0.0 & 0.0 & 0.0 & 0.1 & 0.0 & 0.0 & 0.0 & 0.2 & $* * *$ & $\nearrow$ \\
\hline 69.003 & & & 0.0 & 0.0 & 0.0 & 0.0 & 0.1 & 0.1 & 0.0 & 1.0 & $* * *$ & $\nearrow$ \\
\hline 69.033 & $\mathrm{C} 4 \mathrm{H} 4 \mathrm{OH}+$ & Furan & 0.8 & 0.2 & 0.2 & 1.9 & 2.8 & 3.1 & 0.3 & 34.0 & $* * *$ & $\nearrow$ \\
\hline 69.056 & & & 11.8 & 6.7 & 0.5 & 52.6 & 22.6 & 12.9 & 1.6 & 117.0 & $* * *$ & $\nearrow$ \\
\hline
\end{tabular}


Table 1 continued

\begin{tabular}{|c|c|c|c|c|c|c|c|c|c|c|c|c|}
\hline \multirow{2}{*}{$m / z$} & \multirow[t]{2}{*}{ Formula } & \multirow[t]{2}{*}{ Tentative identification } & \multicolumn{4}{|l|}{ Raw } & \multicolumn{4}{|c|}{ Roasted } & \multirow[t]{2}{*}{$P$ value } & \multirow[t]{2}{*}{ Variation } \\
\hline & & & mean & std & $\min$ & $\max$ & mean & std & $\min$ & $\max$ & & \\
\hline 69.071 & $\mathrm{C} 5 \mathrm{H} 9+$ & Isoprene, common fragment & 5.7 & 3.1 & 1.5 & 18.3 & 22.0 & 40.1 & 0.4 & 549.3 & $* * *$ & $\nearrow$ \\
\hline 71.051 & $\mathrm{C} 4 \mathrm{H} 6 \mathrm{OH}+$ & Butenal & 1.2 & 0.5 & 0.6 & 5.8 & 3.8 & 4.8 & 0.8 & 62.9 & $* * *$ & $\nearrow$ \\
\hline 71.086 & $\mathrm{C} 5 \mathrm{H} 11+$ & $\begin{array}{l}\text { 2-Pentanol, 2-Methyl-1-butanol+3- } \\
\text { Methyl-1-butanol, Pentanol }\end{array}$ & 29.2 & 21.9 & 5.2 & 188.4 & 14.7 & 12.3 & 3.2 & 106.5 & $* * *$ & $\bigcirc$ \\
\hline 72.962 & & & 0.0 & 0.0 & 0.0 & 0.0 & 0.1 & 0.2 & 0.0 & 1.1 & $* * *$ & $\nearrow$ \\
\hline 73.029 & $\mathrm{C} 3 \mathrm{H} 4 \mathrm{O} 2 \mathrm{H}+$ & Propiolactone, Propenoic acid & 1.4 & 0.3 & 0.7 & 2.3 & 2.0 & 1.0 & 0.1 & 6.7 & $* * *$ & $\nearrow$ \\
\hline 73.051 & & & 13.1 & 5.9 & 0.7 & 33.4 & 10.1 & 10.5 & 0.0 & 121.3 & $* * *$ & $\bigcirc$ \\
\hline 73.064 & $\mathrm{C} 4 \mathrm{H} 8 \mathrm{OH}+$ & 2-Methyl-Propanal & 19.2 & 13.5 & 5.5 & 127.1 & 141.8 & 220.7 & 8.3 & 1530.9 & $* * *$ & $\nearrow$ \\
\hline 75.035 & & & 0.4 & 0.1 & 0.2 & 0.7 & 2.6 & 1.2 & 0.0 & 8.4 & $* * *$ & $\nearrow$ \\
\hline 75.045 & $\mathrm{C} 3 \mathrm{H} 6 \mathrm{O} 2 \mathrm{H}+$ & 1-Hydroxy-2-Propanone & 8.0 & 4.3 & 2.4 & 26.9 & 15.8 & 10.7 & 2.8 & 76.4 & $* * *$ & $\nearrow$ \\
\hline 75.072 & & & 0.6 & 0.4 & 0.1 & 3.9 & 0.9 & 0.9 & 0.1 & 6.6 & $* * *$ & $\nearrow$ \\
\hline 76.954 & $\mathrm{CS} 2 \mathrm{H}+$ & Carbon disulfide & 0.1 & 0.1 & 0.0 & 0.5 & 0.0 & 0.0 & 0.0 & 0.3 & $* * *$ & $\bigcirc$ \\
\hline 77.008 & & & 0.0 & 0.0 & 0.0 & 0.0 & 0.1 & 0.1 & 0.0 & 0.6 & $* * *$ & $\nearrow$ \\
\hline 77.037 & & & 0.2 & 0.2 & 0.1 & 2.4 & 0.4 & 0.6 & 0.0 & 5.3 & $* * *$ & $\nearrow$ \\
\hline 79.040 & $\mathrm{C} 2 \mathrm{H} 6 \mathrm{O} 3 \mathrm{H}+$ & Cluster of ms61.028 & 34.5 & 16.8 & 5.0 & 109.5 & 34.6 & 24.0 & 3.8 & 161.3 & NS & - \\
\hline 79.060 & $\mathrm{C} 6 \mathrm{H} 7+$ & Benzene, aromatic ring fragment & 4.2 & 5.8 & 0.7 & 67.2 & 5.7 & 40.8 & 0.0 & 613.2 & NS & - \\
\hline 79.078 & & & 3.1 & 2.7 & 0.5 & 22.2 & 2.5 & 3.5 & 0.0 & 30.6 & * & ○ \\
\hline 80.060 & & & 0.3 & 0.8 & 0.0 & 10.5 & 0.3 & 0.9 & 0.0 & 7.9 & NS & - \\
\hline 81.041 & $\mathrm{C} 4 \mathrm{H} 4 \mathrm{~N} 2 \mathrm{H}+$ & Pyrazine & 1.1 & 0.7 & 0.2 & 8.4 & 1.1 & 0.9 & 0.2 & 8.1 & NS & - \\
\hline 81.070 & $\mathrm{C} 6 \mathrm{H} 9+$ & & 2.6 & 1.7 & 0.9 & 13.1 & 1.2 & 0.9 & 0.4 & 9.7 & $* * *$ & $\bigcirc$ \\
\hline 83.051 & $\mathrm{C} 5 \mathrm{H} 6 \mathrm{OH}+$ & Methylfuran & 3.4 & 1.8 & 0.6 & 17.5 & 4.1 & 1.9 & 0.8 & 16.6 & $* * *$ & $\nearrow$ \\
\hline 83.076 & & & 7.2 & 7.4 & 0.5 & 61.2 & 2.8 & 4.3 & 0.0 & 30.7 & $* * *$ & ○ \\
\hline 83.087 & $\mathrm{C} 6 \mathrm{H} 11+$ & Hexenol, Hexanal & 1.7 & 1.9 & 0.0 & 15.5 & 9.3 & 34.5 & 0.0 & 466.1 & $* * *$ & $\nearrow$ \\
\hline 84.087 & & & 0.7 & 2.3 & 0.1 & 27.6 & 0.0 & 0.0 & 0.0 & 0.0 & $* * *$ & $\bigcirc$ \\
\hline 85.030 & & & 0.0 & 0.0 & 0.0 & 0.0 & 0.6 & 0.3 & 0.3 & 3.4 & $* * *$ & $\nearrow$ \\
\hline 85.067 & $\mathrm{C} 5 \mathrm{H} 8 \mathrm{OH}+$ & Pentanal, Pentanone & 1.0 & 0.8 & 0.4 & 8.3 & 2.4 & 1.7 & 0.5 & 12.3 & $* * *$ & $\nearrow$ \\
\hline 85.102 & $\mathrm{C} 6 \mathrm{H} 13+$ & Hexanol & 13.1 & 20.5 & 0.5 & 220.2 & 5.2 & 11.2 & 0.2 & 128.2 & $* * *$ & $\bigcirc$ \\
\hline 86.009 & & & 0.0 & 0.0 & 0.0 & 0.0 & 0.2 & 0.1 & 0.0 & 1.6 & $* * *$ & $\nearrow$ \\
\hline 87.045 & $\mathrm{C} 4 \mathrm{H} 6 \mathrm{O} 2 \mathrm{H}+$ & Y-Butyrolactone & 5.4 & 2.9 & 1.7 & 23.1 & 13.4 & 12.0 & 2.7 & 114.6 & $* * *$ & $\nearrow$ \\
\hline 87.081 & $\mathrm{C} 5 \mathrm{H} 10 \mathrm{OH}+$ & $\begin{array}{l}\text { 2-Methyl Butanal, 3-Methyl Butanal, 2- } \\
\text { Pentanone }\end{array}$ & 4.8 & 14.7 & 0.9 & 268.5 & 29.5 & 47.7 & 1.9 & 566.8 & $* * *$ & $\nearrow$ \\
\hline 89.061 & $\mathrm{C} 4 \mathrm{H} 8 \mathrm{O} 2 \mathrm{H}+$ & Ethyl acetate & 3.4 & 2.7 & 1.0 & 23.6 & 6.7 & 13.4 & 0.8 & 164.3 & $* * *$ & $\nearrow$ \\
\hline 91.057 & $\mathrm{C} 4 \mathrm{H} 10 \mathrm{SH}+$ & Diethyl sulfide & 2.4 & 3.8 & 0.6 & 41.8 & 3.2 & 7.0 & 0.6 & 67.2 & * & $\nearrow$ \\
\hline 91.075 & $\mathrm{C} 4 \mathrm{H} 10 \mathrm{O} 2 \mathrm{H}+$ & Butanediol & 1.2 & 0.9 & 0.2 & 8.5 & 4.7 & 7.5 & 0.0 & 58.0 & $* * *$ & $\nearrow$ \\
\hline 93.040 & & & 0.0 & 0.0 & 0.0 & 0.0 & 6.5 & 1.8 & 3.2 & 14.2 & $* * *$ & $\nearrow$ \\
\hline 93.073 & $\mathrm{C} 7 \mathrm{H} 9+$ & Toluene & 2.4 & 1.2 & 0.9 & 9.3 & 1.5 & 0.9 & 0.4 & 4.9 & $* * *$ & $\bigcirc$ \\
\hline 93.091 & & & 4.4 & 9.2 & 0.2 & 110.1 & 1.6 & 5.2 & 0.0 & 56.9 & $* * *$ & $\bigcirc$ \\
\hline 95.051 & $\mathrm{C} 6 \mathrm{H} 6 \mathrm{OH}+$ & Phenol & 1.8 & 1.4 & 1.1 & 18.8 & 2.4 & 5.1 & 1.1 & 47.7 & * & $\nearrow$ \\
\hline
\end{tabular}


Table 1 continued

\begin{tabular}{|c|c|c|c|c|c|c|c|c|c|c|c|c|}
\hline \multirow[t]{2}{*}{$m / z$} & \multirow[t]{2}{*}{ Formula } & \multirow[t]{2}{*}{ Tentative identification } & \multicolumn{4}{|l|}{ Raw } & \multicolumn{4}{|c|}{ Roasted } & \multirow[t]{2}{*}{$P$ value } & \multirow[t]{2}{*}{ Variation } \\
\hline & & & mean & std & $\min$ & $\max$ & mean & std & $\min$ & $\max$ & & \\
\hline 95.088 & $\mathrm{C} 7 \mathrm{H} 11+$ & Heptenal & 0.7 & 0.6 & 0.3 & 8.5 & 0.5 & 0.5 & 0.0 & 5.9 & $* * *$ & 0 \\
\hline 97.048 & $\mathrm{C} 5 \mathrm{H} 4 \mathrm{O} 2 \mathrm{H}+$ & Furfural & 0.7 & 0.3 & 0.2 & 1.8 & 2.7 & 5.9 & 0.4 & 61.6 & $* * *$ & $\nearrow$ \\
\hline 97.066 & $\mathrm{C} 6 \mathrm{H} 8 \mathrm{OH}+$ & Ethylfuran & 0.3 & 0.1 & 0.2 & 0.7 & 1.0 & 0.5 & 0.3 & 4.6 & $* * *$ & $\nearrow$ \\
\hline 97.102 & $\mathrm{C} 7 \mathrm{H} 13+$ & Heptanal & 0.5 & 0.7 & 0.2 & 8.0 & 0.3 & 0.6 & 0.1 & 7.9 & $* * *$ & ○ \\
\hline 99.046 & $\mathrm{C} 5 \mathrm{H} 6 \mathrm{O} 2 \mathrm{H}+$ & 2-Furan methanol & 0.5 & 0.1 & 0.2 & 0.9 & 0.7 & 0.5 & 0.3 & 5.7 & $* * *$ & $\nearrow$ \\
\hline 99.082 & $\mathrm{C} 6 \mathrm{H} 10 \mathrm{OH}+$ & Hexenal & 0.4 & 0.2 & 0.2 & 1.5 & 0.6 & 0.3 & 0.3 & 4.6 & $* * *$ & $\nearrow$ \\
\hline 99.117 & $\mathrm{C} 7 \mathrm{H} 15+$ & Heptanol & 0.7 & 1.3 & 0.0 & 23.6 & 0.2 & 0.5 & 0.0 & 9.2 & $* * *$ & $\bigcirc$ \\
\hline 99.951 & & & 0.0 & 0.0 & 0.0 & 0.0 & 0.0 & 0.0 & 0.0 & 0.1 & $* * *$ & $\nearrow$ \\
\hline 101.062 & $\mathrm{C} 5 \mathrm{H} 8 \mathrm{O} 2 \mathrm{H}+$ & 2,3-Pentanedione & 1.4 & 0.6 & 0.5 & 4.9 & 2.1 & 1.5 & 0.6 & 12.6 & $* * *$ & $\nearrow$ \\
\hline 101.097 & $\mathrm{C} 6 \mathrm{H} 12 \mathrm{OH}+$ & Hexanal & 2.5 & 10.6 & 0.2 & 131.8 & 3.4 & 11.3 & 0.2 & 143.5 & NS & - \\
\hline 103.052 & & & 0.2 & 0.1 & 0.1 & 1.0 & 0.6 & 0.7 & 0.1 & 6.4 & $* * *$ & $\nearrow$ \\
\hline 103.077 & $\mathrm{C} 5 \mathrm{H} 10 \mathrm{O} 2 \mathrm{H}+$ & C5 esters and acids & 0.6 & 0.3 & 0.2 & 4.3 & 0.6 & 0.4 & 0.2 & 3.6 & NS & - \\
\hline 103.115 & & & 0.1 & 0.2 & 0.0 & 2.9 & 0.0 & 0.1 & 0.0 & 1.2 & $* * *$ & 0 \\
\hline 105.039 & $\mathrm{C} 4 \mathrm{H} 8 \mathrm{OSH}+$ & Methional & 0.4 & 0.3 & 0.0 & 2.4 & 0.6 & 1.9 & 0.1 & 16.1 & $* *$ & $\nearrow$ \\
\hline 105.068 & $\mathrm{C} 8 \mathrm{H} 9+$ & Phenyl ethyl alcohol & 2.7 & 4.7 & 0.4 & 44.0 & 1.5 & 1.9 & 0.0 & 16.9 & $* * *$ & ○ \\
\hline 105.090 & $\mathrm{C} 5 \mathrm{H} 12 \mathrm{O} 2 \mathrm{H}+$ & & 0.0 & 0.0 & 0.0 & 0.0 & 2.4 & 3.6 & 0.1 & 40.2 & $* * *$ & $\nearrow$ \\
\hline 107.044 & $\mathrm{C} 7 \mathrm{H} 6 \mathrm{OH}+$ & Benzaldehyde & 15.4 & 70.0 & 0.3 & 955.3 & 13.5 & 93.4 & 0.3 & 1119.1 & NS & - \\
\hline 107.089 & $\mathrm{C} 8 \mathrm{H} 11+$ & $\begin{array}{l}\text { Ethyl benzene, p-Xylene, m-Xylene, o- } \\
\text { Xylene }\end{array}$ & 2.3 & 4.4 & 0.5 & 52.9 & 1.5 & 6.9 & 0.0 & 118.0 & NS & - \\
\hline 109.065 & $\mathrm{C} 7 \mathrm{H} 8 \mathrm{OH}+$ & Benzyl alcohol, Cresol & 0.3 & 0.3 & 0.1 & 3.2 & 0.4 & 0.9 & 0.1 & 7.5 & $* *$ & $\nearrow$ \\
\hline 109.103 & $\mathrm{C} 8 \mathrm{H} 13+$ & Octenal & 0.5 & 0.4 & 0.2 & 4.9 & 0.5 & 0.7 & 0.0 & 8.4 & NS & - \\
\hline 111.047 & $\mathrm{C} 6 \mathrm{H} 6 \mathrm{O} 2 \mathrm{H}+$ & & 2.3 & 0.6 & 0.5 & 3.8 & 1.8 & 0.9 & 0.5 & 9.1 & $* * *$ & ○ \\
\hline 111.084 & & & 0.3 & 0.1 & 0.2 & 0.7 & 0.5 & 0.3 & 0.1 & 2.1 & $* * *$ & $\nearrow$ \\
\hline 111.118 & $\mathrm{C} 8 \mathrm{H} 15+$ & 1-Octen-3-ol & 0.5 & 0.8 & 0.1 & 11.2 & 0.3 & 0.4 & 0.1 & 6.9 & $* * *$ & ○ \\
\hline 113.064 & $\mathrm{C} 6 \mathrm{H} 8 \mathrm{O} 2 \mathrm{H}+$ & 2(5H)-Furanone, 5,5-dimethyl- & 0.3 & 0.1 & 0.2 & 0.5 & 0.2 & 0.1 & 0.1 & 0.6 & $* * *$ & ○ \\
\hline 113.099 & $\mathrm{C} 7 \mathrm{H} 12 \mathrm{OH}+$ & Heptanal, Heptanone & 0.1 & 0.1 & 0.1 & 1.7 & 0.3 & 0.5 & 0.1 & 5.5 & $* * *$ & $\nearrow$ \\
\hline 113.133 & $\mathrm{C} 8 \mathrm{H} 17+$ & 2-Ethyl-1-Hexanol, Octanol & 1.0 & 0.5 & 0.1 & 2.4 & 0.2 & 0.1 & 0.0 & 0.9 & $* * *$ & ○ \\
\hline 115.078 & $\mathrm{C} 6 \mathrm{H} 10 \mathrm{O} 2 \mathrm{H}+$ & Caprolactone & 0.5 & 0.7 & 0.2 & 12.5 & 0.5 & 0.5 & 0.2 & 8.2 & NS & - \\
\hline 115.114 & $\mathrm{C} 7 \mathrm{H} 14 \mathrm{OH}+$ & 2-Heptanal (E o Z) & 0.6 & 2.4 & 0.1 & 38.8 & 0.4 & 1.3 & 0.1 & 19.2 & NS & - \\
\hline 117.062 & $\mathrm{C} 9 \mathrm{H} 9+$ & & 0.2 & 0.1 & 0.0 & 0.5 & 0.2 & 0.1 & 0.1 & 0.5 & $* * *$ & ○ \\
\hline 117.092 & $\mathrm{C} 6 \mathrm{H} 12 \mathrm{O} 2 \mathrm{H}+$ & $\begin{array}{l}\text { Butanoic acid ethyl ester, butyl } \\
\text { acetate, Hexanoic acid }\end{array}$ & 0.5 & 1.0 & 0.1 & 18.5 & 0.6 & 0.7 & 0.1 & 12.1 & NS & - \\
\hline 117.958 & & & 0.0 & 0.0 & 0.0 & 0.0 & 0.0 & 0.0 & 0.0 & 0.4 & $* * *$ & $\nearrow$ \\
\hline 119.106 & $\mathrm{C} 6 \mathrm{H} 14 \mathrm{O} 2 \mathrm{H}+$ & & 0.5 & 2.4 & 0.0 & 29.4 & 0.7 & 1.9 & 0.0 & 23.7 & NS & - \\
\hline 121.067 & $\mathrm{C} 8 \mathrm{H} 8 \mathrm{OH}+$ & Benzeneacetaldehyde & 0.4 & 0.3 & 0.2 & 4.8 & 1.9 & 1.9 & 0.3 & 23.9 & $* * *$ & $\nearrow$ \\
\hline 121.104 & $\mathrm{C9H13+}$ & & 0.2 & 0.1 & 0.1 & 1.1 & 0.1 & 0.1 & 0.0 & 0.4 & $* * *$ & 0 \\
\hline 121.120 & & & 0.2 & 0.4 & 0.0 & 4.4 & 0.1 & 0.1 & 0.0 & 1.1 & $* * *$ & ○ \\
\hline 123.048 & & & 0.2 & 0.0 & 0.1 & 0.3 & 0.2 & 0.1 & 0.1 & 0.4 & $* * *$ & 0 \\
\hline
\end{tabular}


Table 1 continued

\begin{tabular}{|c|c|c|c|c|c|c|c|c|c|c|c|c|}
\hline \multirow[t]{2}{*}{$m / z$} & \multirow[t]{2}{*}{ Formula } & \multirow[t]{2}{*}{ Tentative identification } & \multicolumn{4}{|l|}{ Raw } & \multicolumn{4}{|c|}{ Roasted } & \multirow[t]{2}{*}{$P$ value } & \multirow[t]{2}{*}{ Variation } \\
\hline & & & mean & std & $\min$ & $\max$ & mean & std & $\min$ & $\max$ & & \\
\hline 123.118 & $\mathrm{C} 9 \mathrm{H} 15+$ & Nonenal & 0.1 & 0.1 & 0.1 & 0.7 & 0.1 & 0.1 & 0.1 & 0.7 & * & ○ \\
\hline 125.059 & $\mathrm{C} 7 \mathrm{H} 8 \mathrm{O} 2 \mathrm{H}+$ & Benzyl alcohol & 0.6 & 0.9 & 0.1 & 7.0 & 0.4 & 1.3 & 0.1 & 9.6 & NS & - \\
\hline 125.100 & $\mathrm{C} 8 \mathrm{H} 12 \mathrm{OH}+$ & Octadienone & 0.2 & 0.2 & 0.0 & 1.9 & 0.2 & 0.3 & 0.0 & 2.6 & NS & - \\
\hline 125.134 & $\mathrm{C} 9 \mathrm{H} 17+$ & Nonanal, Nonenol & 0.3 & 0.2 & 0.1 & 2.4 & 0.1 & 0.1 & 0.0 & 1.4 & $* * *$ & $\bigcirc$ \\
\hline 127.042 & $\mathrm{C} 6 \mathrm{H} 6 \mathrm{O} 3 \mathrm{H}+$ & Maltol & 0.0 & 0.0 & 0.0 & 0.1 & 0.1 & 0.1 & 0.0 & 0.7 & $* * *$ & $\nearrow$ \\
\hline 127.075 & $\mathrm{C} 7 \mathrm{H} 10 \mathrm{O} 2 \mathrm{H}+$ & & 0.1 & 0.0 & 0.1 & 0.3 & 0.1 & 0.0 & 0.0 & 0.5 & $* * *$ & $\bigcirc$ \\
\hline 127.114 & $\mathrm{C} 8 \mathrm{H} 14 \mathrm{OH}+$ & 6-Methyl-5-Hepten-2-one & 0.2 & 0.3 & 0.1 & 5.5 & 0.3 & 0.6 & 0.0 & 8.3 & NS & - \\
\hline 127.148 & $\mathrm{C9H19+}$ & Nonanol & 1.6 & 0.9 & 0.0 & 6.3 & 0.2 & 0.2 & 0.0 & 2.2 & $* * *$ & $\bigcirc$ \\
\hline 129.056 & $\mathrm{C} 6 \mathrm{H} 8 \mathrm{O} 3 \mathrm{H}+$ & Furaneol & 0.2 & 0.0 & 0.1 & 0.3 & 0.2 & 0.1 & 0.1 & 0.4 & NS & - \\
\hline 129.094 & $\mathrm{C} 7 \mathrm{H} 12 \mathrm{O} 2 \mathrm{H}+$ & & 0.1 & 0.0 & 0.1 & 0.6 & 0.1 & 0.1 & 0.1 & 0.7 & NS & - \\
\hline 129.129 & $\mathrm{C} 8 \mathrm{H} 16 \mathrm{OH}+$ & Octanal & 0.5 & 1.2 & 0.1 & 12.6 & 0.3 & 0.9 & 0.0 & 9.6 & * & ○ \\
\hline 131.108 & $\mathrm{C} 7 \mathrm{H} 14 \mathrm{O} 2 \mathrm{H}+$ & Isoamyl acetate & 0.1 & 0.1 & 0.0 & 1.2 & 0.1 & 0.1 & 0.0 & 1.3 & NS & \\
\hline 133.121 & $\mathrm{C} 7 \mathrm{H} 16 \mathrm{O} 2 \mathrm{H}+$ & & 0.1 & 0.1 & 0.0 & 1.9 & 0.1 & 0.2 & 0.0 & 1.7 & NS & - \\
\hline 134.973 & & & 0.0 & 0.0 & 0.0 & 0.2 & 0.0 & 0.0 & 0.0 & 0.2 & $* * *$ & $\nearrow$ \\
\hline 135.120 & $\mathrm{C} 10 \mathrm{H} 15+$ & Cymene & 0.1 & 0.1 & 0.0 & 1.1 & 0.1 & 0.1 & 0.0 & 0.9 & $* * *$ & ○ \\
\hline 136.993 & & & 0.1 & 0.1 & 0.0 & 0.5 & 0.1 & 0.1 & 0.0 & 0.4 & $* * *$ & ○ \\
\hline 137.134 & $\mathrm{C} 10 \mathrm{H} 17+$ & Limonene & 0.9 & 0.7 & 0.2 & 5.3 & 0.3 & 0.2 & 0.1 & 1.7 & $* * *$ & ○ \\
\hline 139.114 & $\mathrm{C} 9 \mathrm{H} 14 \mathrm{OH}+$ & 2-Pentyl Furan & 0.3 & 0.5 & 0.1 & 5.2 & 0.5 & 1.2 & 0.0 & 12.2 & $* * *$ & $\nearrow$ \\
\hline 141.130 & $\mathrm{C} 9 \mathrm{H} 16 \mathrm{OH}+$ & Nonenal, Nonenone & 0.1 & 0.1 & 0.0 & 0.6 & 0.1 & 0.1 & 0.0 & 0.9 & NS & - \\
\hline 143.110 & $\mathrm{C} 8 \mathrm{H} 14 \mathrm{O} 2 \mathrm{H}+$ & & 0.2 & 0.2 & 0.1 & 3.8 & 0.2 & 0.2 & 0.1 & 2.5 & NS & - \\
\hline 143.145 & $\mathrm{C} 9 \mathrm{H} 18 \mathrm{OH}+$ & Nonanal & 0.8 & 1.1 & 0.1 & 18.0 & 0.4 & 0.6 & 0.1 & 8.0 & $* * *$ & ○ \\
\hline 145.123 & $\mathrm{C} 8 \mathrm{H} 16 \mathrm{O} 2 \mathrm{H}+$ & Hexyl acetate & 0.1 & 0.1 & 0.0 & 0.7 & 0.1 & 0.1 & 0.0 & 0.9 & $* *$ & $\nearrow$ \\
\hline 147.137 & $\mathrm{C} 8 \mathrm{H} 18 \mathrm{O} 2 \mathrm{H}+$ & & 0.0 & 0.1 & 0.0 & 1.4 & 0.0 & 0.1 & 0.0 & 0.8 & NS & - \\
\hline 149.059 & $\mathrm{C} 9 \mathrm{H} 8 \mathrm{O} 2 \mathrm{H}+$ & Cinnamic acid & 0.0 & 0.0 & 0.0 & 0.0 & 0.1 & 0.1 & 0.0 & 1.1 & $* * *$ & $\nearrow$ \\
\hline 149.119 & $\mathrm{C} 7 \mathrm{H} 16 \mathrm{O} 3 \mathrm{H}+$ & & 0.1 & 0.1 & 0.0 & 1.3 & 0.0 & 0.1 & 0.0 & 0.8 & $* * *$ & $\bigcirc$ \\
\hline 153.092 & $\mathrm{C} 9 \mathrm{H} 12 \mathrm{O} 2 \mathrm{H}+$ & & 0.0 & 0.0 & 0.0 & 0.0 & 0.0 & 0.0 & 0.0 & 0.3 & $* * *$ & $\nearrow$ \\
\hline 153.131 & $\mathrm{C} 10 \mathrm{H} 16 \mathrm{OH}+$ & & 0.1 & 0.0 & 0.0 & 0.3 & 0.0 & 0.0 & 0.0 & 0.3 & $* * *$ & $\bigcirc$ \\
\hline 155.003 & & & 0.0 & 0.0 & 0.0 & 0.2 & 0.0 & 0.0 & 0.0 & 0.1 & $* * *$ & ○ \\
\hline 155.178 & $\mathrm{C} 11 \mathrm{H} 23+$ & Undecanol & 0.6 & 0.3 & 0.0 & 1.6 & 0.1 & 0.1 & 0.0 & 0.6 & $* * *$ & $\bigcirc$ \\
\hline 157.123 & $\mathrm{C} 9 \mathrm{H} 16 \mathrm{O} 2 \mathrm{H}+$ & Whiskey lactone & 0.1 & 0.0 & 0.0 & 0.3 & 0.1 & 0.0 & 0.0 & 0.3 & $* * *$ & $\bigcirc$ \\
\hline 157.161 & $\mathrm{C} 10 \mathrm{H} 20 \mathrm{OH}+$ & Decanal & 0.1 & 0.1 & 0.0 & 0.7 & 0.1 & 0.0 & 0.0 & 0.5 & $* * *$ & $\bigcirc$ \\
\hline 159.139 & $\mathrm{C9H} 18 \mathrm{O} 2 \mathrm{H}+$ & C9 esters and acids & 0.1 & 0.3 & 0.0 & 5.7 & 0.1 & 0.1 & 0.0 & 1.6 & NS & - \\
\hline 161.151 & $\mathrm{C} 9 \mathrm{H} 20 \mathrm{O} 2 \mathrm{H}+$ & & 0.1 & 0.1 & 0.0 & 1.2 & 0.0 & 0.0 & 0.0 & 0.4 & $* * *$ & $\bigcirc$ \\
\hline 163.132 & $\mathrm{C} 8 \mathrm{H} 18 \mathrm{O} 3 \mathrm{H}+$ & & 0.0 & 0.0 & 0.0 & 0.2 & 0.0 & 0.0 & 0.0 & 0.1 & $* *$ & $\bigcirc$ \\
\hline 165.079 & & & 0.0 & 0.0 & 0.0 & 0.3 & 0.0 & 0.0 & 0.0 & 0.5 & $* * *$ & $\bigcirc$ \\
\hline 167.056 & & & 0.2 & 0.1 & 0.1 & 1.2 & 0.2 & 0.2 & 0.1 & 2.1 & $* * *$ & ○ \\
\hline 169.194 & $\mathrm{C} 12 \mathrm{H} 25+$ & Dodecanol & 0.4 & 0.2 & 0.0 & 1.2 & 0.1 & 0.0 & 0.0 & 0.2 & $* * *$ & $\bigcirc$ \\
\hline 189.175 & $\mathrm{C} 11 \mathrm{H} 24 \mathrm{O} 2 \mathrm{H}+$ & & 0.0 & 0.0 & 0.0 & 0.1 & 0.0 & 0.0 & 0.0 & 0.0 & $* * *$ & ○ \\
\hline
\end{tabular}


Table 1 continued

\begin{tabular}{|c|c|c|c|c|c|c|c|c|c|c|c|c|}
\hline \multirow[t]{2}{*}{$m / z$} & \multirow[t]{2}{*}{ Formula } & \multirow[t]{2}{*}{ Tentative identification } & \multicolumn{4}{|l|}{ Raw } & \multicolumn{4}{|c|}{ Roasted } & \multirow[t]{2}{*}{$P$ value } & \multirow[t]{2}{*}{ Variation } \\
\hline & & & mean & std & $\min$ & $\max$ & mean & std & $\min$ & $\max$ & & \\
\hline 197.086 & & & 0.0 & 0.0 & 0.0 & 0.0 & 0.0 & 0.0 & 0.0 & 0.0 & $* * *$ & $\nearrow$ \\
\hline 205.197 & $\mathrm{C} 15 \mathrm{H} 25+$ & Sesquiterpenes & 0.1 & 0.3 & 0.0 & 4.8 & 0.0 & 0.2 & 0.0 & 3.2 & NS & - \\
\hline 223.066 & & & 1.3 & 5.1 & 0.1 & 56.7 & 1.2 & 4.4 & 0.1 & 57.0 & NS & - \\
\hline
\end{tabular}

Each VOC mass peak was tentatively identified based on an in-house library of pure standards and on literature review. VOC mass peak values were reported as concentration $\left(\mathrm{ppb}_{\mathrm{v}}\right)$. The average $(n=3)$, standard deviation, minimum and maximum values were reported

${ }^{*} p$ value $<0.05 ;{ }^{* *} p$ value $<0.01 ;{ }^{* * *} p$ value $<0.001$; $\nearrow$ increase during roasting; \decrease during roasting

encompasses accessions showing both high resistance/ tolerance to biotic and abiotic stresses and/or quality traits of interest. Such a genetic reservoir could play a fundamental role in future breeding plans in which specific traits characterizing local selections could be introgressed into elite cultivars through marker-assisted breeding selection approaches.

In this survey, our almond collection was both phenotyped using a proton-transfer time-of-flight mass spectrometer and genotyped using the Illumina Infinium ${ }^{\circledR} 18 \mathrm{~K}$ Peach SNP array developed by RosBREED consortium ${ }^{31}$. Genetic data were employed for synteny analysis and to decipher both the genetic stratification and the linkage disequilibrium (LD) extent within the collection in the analysis. The same germplasm was phenotyped for the production of VOCs both on raw and roasted kernels using a PTR-ToF-MS.

The aims of this work were (i) to estimate the volatilome variability among almond different genotypes; (ii) to evaluate the effect of roasting on the VOC composition of the almond kernel; (iii) to identify the best performing accessions to be used as superior parental lines for future breeding programs; (iv) to detect molecular markers linked to VOCs of interest. In addition, the results of this study might be useful in defining an objective VOC phenotyping protocol to apply in all production stages, from breeding to the food industry transformation. This study is a first, preliminary, step toward the definition of molecular markers that can be readily employed for marker-assisted selection (MAS) approaches and provide novel insights on the genetic mechanisms regulating the VOCs profile in almond.

\section{Results and discussion}

\section{High-resolution VOC phenotyping}

Almond VOC profile was assessed on raw and roasted kernels in triplicate by PTR-ToF-MS analysis as described in Farneti et al. ${ }^{11}$. VOC mass peaks from the raw PTR-ToF-MS spectra were reduced from 422 to 150 , applying noise and correlation coefficient thresholds (Table 1, Supplementary
Fig. 1, Supplementary Tables 1-2). Tentative identification (t.i.) of each mass peak, detected by PTR-ToF-MS, was based on in-house library of pure standards and on literature review $^{32-36}$. VOC profile was considerably altered during roasting, as 122 mass peaks significantly differed between raw and roasted almonds (Table 1, Supplementary Fig. 1, Supplementary Tables 1-2). To our knowledge, this is the first work about PTR-MS application on almond kernels; this technique has already been successfully applied for the characterization of fermented cocoa and green and roasted coffee beans $^{13,14}$ and for the online monitoring of coffee roasting $^{37-39}$.

Among the 150 mass peaks, 68 significantly increased their content during roasting (Table 1, Fig. 1, Supplementary Fig. 1). Most of these masses were related with sulfur compounds, such as $m / z 34.996$ (t.i. hydrogen sulfide), $m / z 49.011$ (t.i. methanethiol), $\mathrm{m} / \mathrm{z} 63.029$ (t.i. dimethyl sulfide, Fig. 1a), $m / z 91.057$ (t.i. diethyl sulfide), and $m / z 105.039$ (t.i. methional), and with furan containing compounds produced by thermal degradation of sugars such as $m / z 69.033$ (t.i. furan), $m / z 83.051$ (t.i. methylfuran), $m / z 97.048$ (t.i. furfural, Fig. 1c), $m / z 97.066$ (t.i. ethylfuran), $m / z 99.046$ (t.i. 2-furan methanol), and $\mathrm{m} / \mathrm{z} 139.114$ (t.i. 2-pentyl furan). Moreover, roasting enhanced the concentration of aldehydes formed by Strecker degradation of valine, isoleucine, leucine and phenylalanine ${ }^{40}$, such as 2-methylpropanal $(\mathrm{m} / \mathrm{z} 73.064)$, 2 - and 3-methylbutanal $(m / z$ 87.081, Fig. $1 \mathrm{~b})$, and benzeneacetaldehyde $(\mathrm{m} / z$ 121.066). Other relevant VOC mass peaks that significantly increased during roasting were methanol $(\mathrm{m} / \mathrm{z} 33,033)$, cyanamide $(\mathrm{m} / \mathrm{z} 43.03)$, acetic acid $(\mathrm{m} / \mathrm{z}$ 61.028), 1-hydroxy-2-propanone $(\mathrm{m} / \mathrm{z}$ 75.044), and $\gamma$-Butyrolactone $(\mathrm{m} / z$ 87,0453) (Table 1$)$. Many of these compounds were found in roasted almonds and other nuts such as hazelnuts ${ }^{41}$, walnuts ${ }^{42}$, pecans ${ }^{43}$, peanuts ${ }^{44}$. Some similarities were also found with the aroma compound formation during coffee roasting which is well studied both by GC-MS and PTR-MS. However, the aroma profile of roasted coffee is usually richer in pyrroles, pyrazines, and other products of Maillard 
a

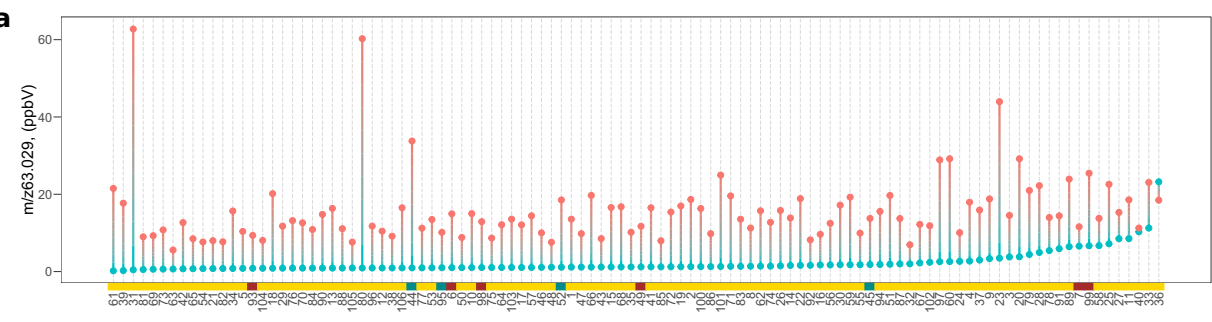

b

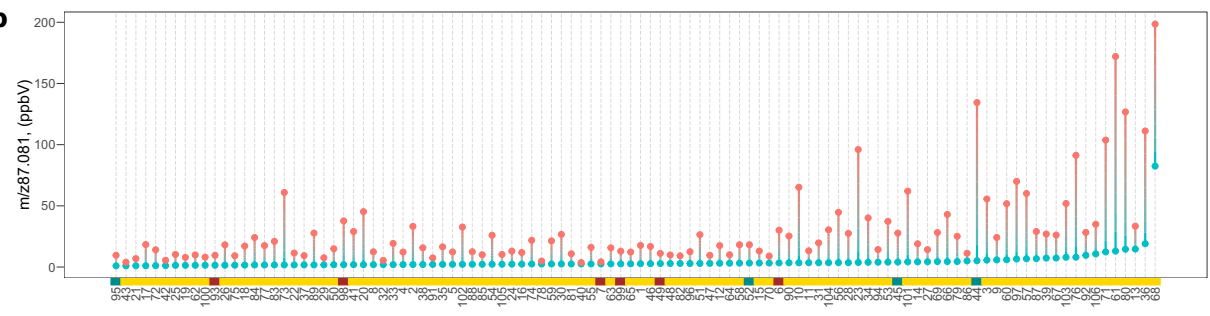

C

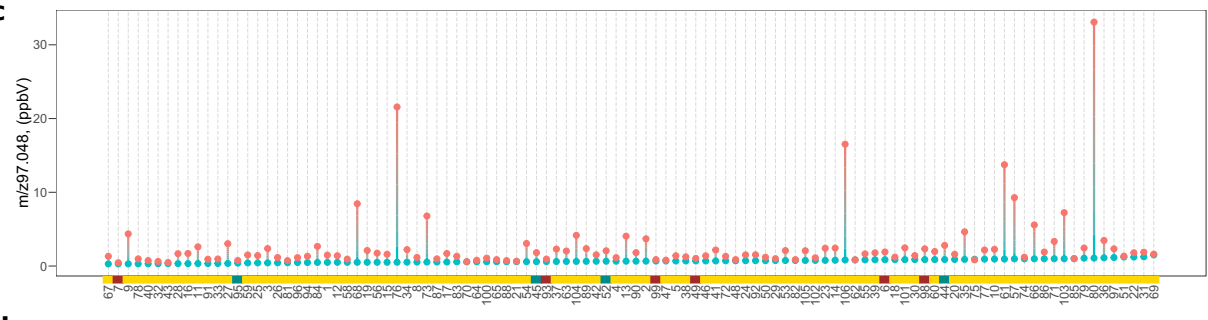

d

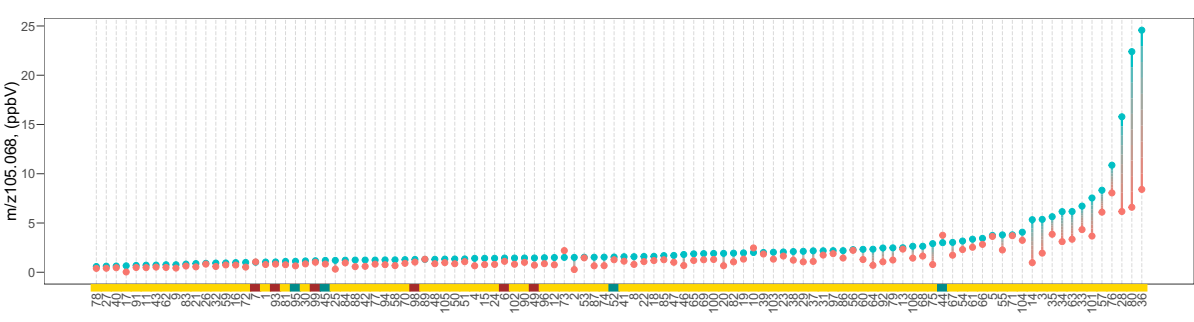

e

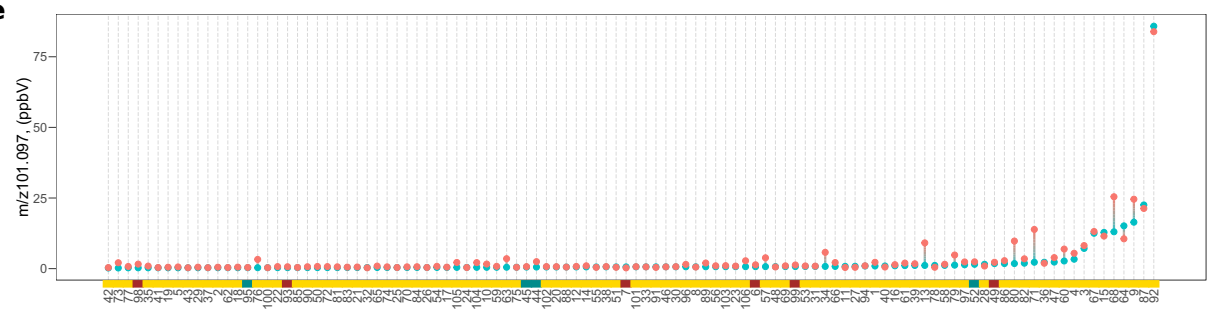

f

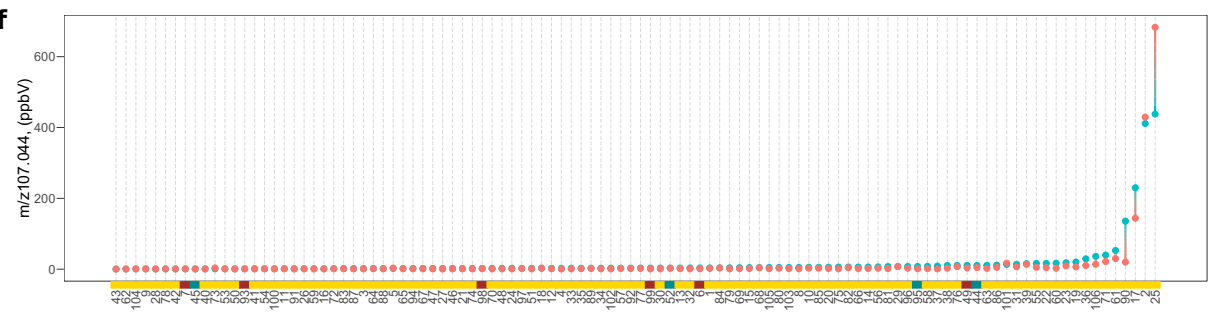

Fig. 1 Lollipop graph of six VOC mass peaks characteristic of almond aroma profile (out of 150 detected in total by proton transfer reaction time of flight mass spectrometer, PTR-ToF-MS). a 63.029 (t.i. dimethyl sulfide), b 87.081 (t.i. 2- and 3-methylbutanal), c 97.048 (t.i. furfural), d 105.068 (t.i. phenyl ethyl alcohol), e 101.097 (t.i. hexanal), f 107.044 (t.i. benzaldehyde). Each graph illustrates the average value of three measurements recorded on fresh (blue) and roasted (pink) almond kernels. The corresponding complete names of the accessions were reported in supplementary table 1. In each graph, accessions were ordered based on the VOC mass peak concentration recorded on the raw kernel. The coloured line below the lollipop graph summarized the origin of each accession (red: Apulia, blue: International, yellow: Sicily). Lollipop graphs, together with violin plots and correlation plots of all 150 VOC mass peaks, are reported in Fig. S1 
a

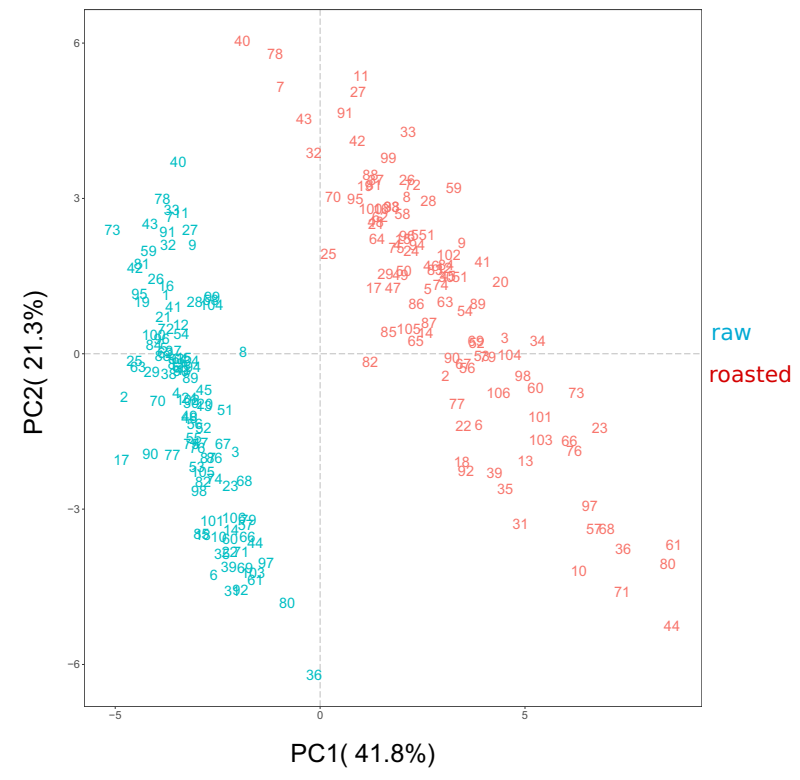

c
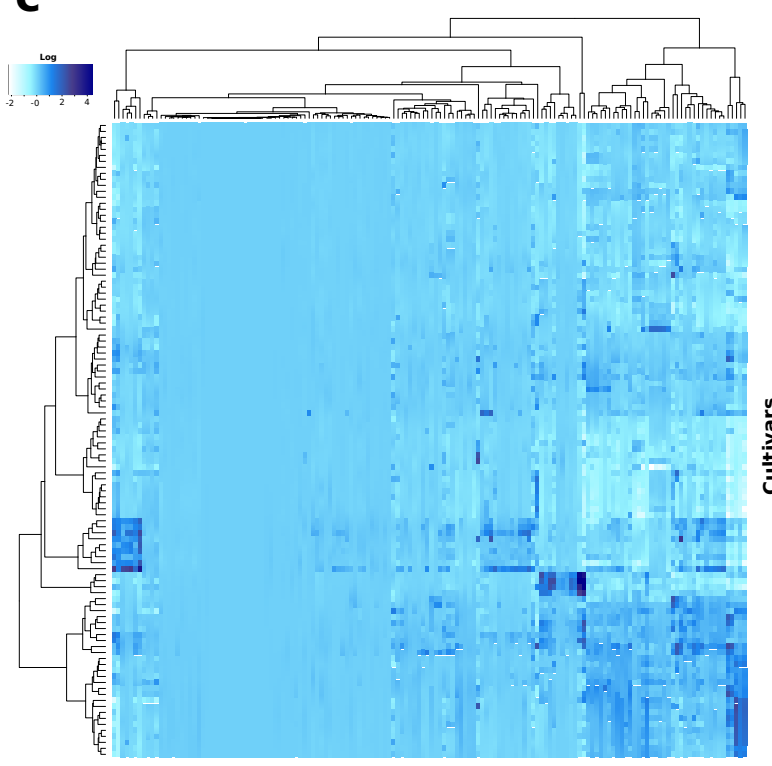

$\operatorname{voCs}(\mathbf{m} / \mathbf{z})$ b

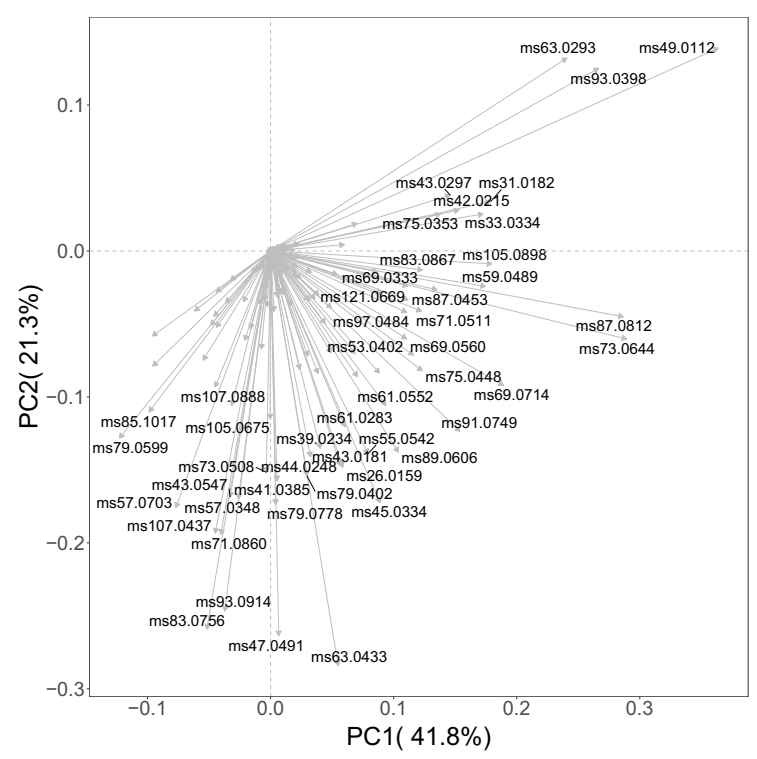

d
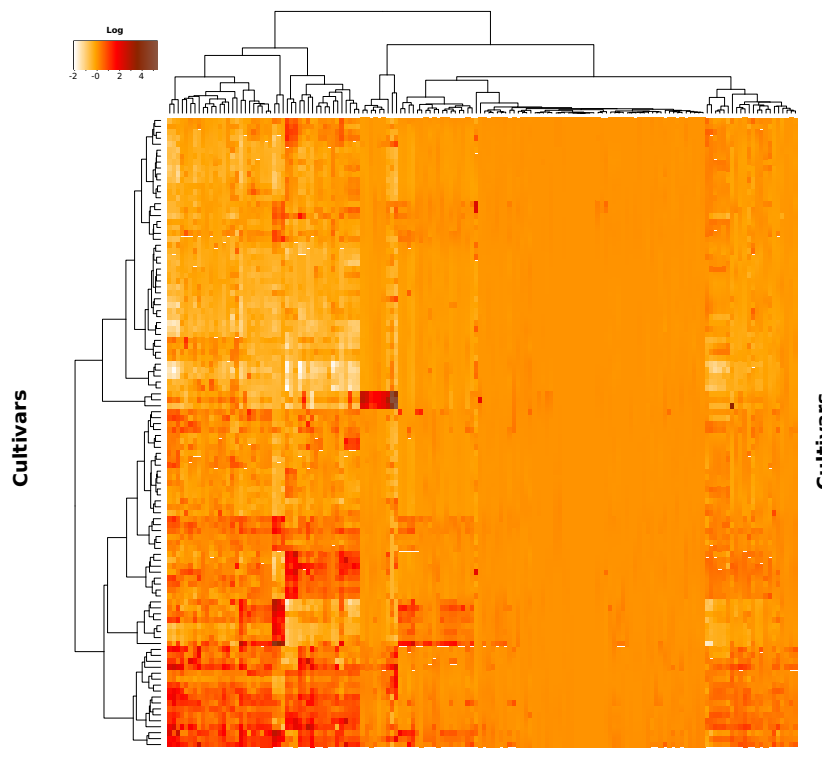

$\operatorname{voCs}(m / z)$

Fig. 2 Almond VOCs assessment. Principal component analysis (PCA) (a), loading plot (b) and heatmaps with two-dimensional hierarchical dendrograms of the fresh (c) and roasted (d) VOC mass peaks detected in the 106 Prunus dulcis accessions assessed by proton transfer reaction time of flight mass spectrometer (PTR-ToF-MS). Each VOC concentration is the average of three biological replicates

reaction, since coffee beans undergo the roasting for longer time and higher temperatures. These compounds were also found in almonds after a longer roasting time (data not shown).

On the contrary, fewer mass peaks (54 over 150) were significantly reduced during roasting (Table 1 ). Many of them were related with alcohol compounds, in particular ethanol $(\mathrm{m} / \mathrm{z} 47.049)$, butanol $(\mathrm{m} / \mathrm{z} 57.07)$, 2-pentanol $(m / z$ 71.086), hexanol $(m / z$ 85.10), phenyl ethyl alcohol $(\mathrm{m} / z$ 105.068, Figs. $1 \mathrm{~d}), 1$-octen-3-ol $(\mathrm{m} / \mathrm{z}$ 111.118), and nonanol $(\mathrm{m} / z$ 127.148). Other relevant VOCs that significantly decreased during roasting were aminoacetonitrile $(m / z 57.043)$ and limonene $(m / z$ 137.13) (Table 1$)$.

Only a few mass peaks (28 over 150) were not significantly modified by roasting. Among them, several compounds have an important role in the characterization 
of almond aroma ${ }^{33,35}$, such as benzaldehyde $(\mathrm{m} / z$ 107.043, Fig. 1f), benzene $(m / z 79.04)$, ethyl benzene $(m / z$ 107.088), pyrazine $(m / z 81.041)$ and hexanal $(m / z$ 101.097, Fig. 1e).

The VOC variability, assessed on raw and roasted almonds, is graphically represented by the PCA plot (Fig. 2a, b) defined by the first two PCs (PC1: $41.8 \%$ and PC2: $21.3 \%$ of the total phenotypic variability). VOC differences related to roasting were mostly explainable by $\mathrm{PC} 1$, while differences among almond genotypes, in particular for fresh kernels, were mostly related to PC2. Cultivars defined by negative values of $\mathrm{PC} 2$ had a more intense VOC profile for both fresh and roasted kernels, as it was also validated by the hierarchical clustering and heatmap (Fig. 2c, d). Almond VOC profile seemed to be mostly influenced by roasting, but still with significant interaction with genetic variability. As a result, fresh and roasted almond genotypes were significantly clustered into two groups (Fig. 2a) based on PC1 variability.

According to solely to the VOC profile assessed on fresh kernels, an accurate prediction of the profile after roasting is quite complex, since several compounds, like sulfur compounds, furans, and few aldehydes, are produced by the degradation of primary metabolites only during roasting (Fig. 1 and Supplementary figure 1). However, based on results of both PCA analysis (Fig. 2a, b) and hierarchical clustering (Fig. 2c, d), most of the accessions considered in this study maintained a comparable topological structure of the cluster's tree (Supplementary Fig. 2). In particular, it was possible to identify two clusters of accessions composed respectively by "Angelica" (\#3), "Baggiana" (\#4), "Belvedere" (\#9), "Cacciatura” (\#15), "Montagna" (\#64), "Mullisa Piccola" (\#67), and "Sarbaggia di Sciascia" (\#87); and by "Amara di Martorana" (\#2), "Calamonaci" (\#17) and "Cesaro 1" (\#25) that maintained their VOC characteristics after roasting. These two clusters were characterized, respectively, by an elevated concentration of $m / z 55.054$ (t.i. butanal), $m / z 83.086$ (t.i. hexenol), $m / z 85.102$ (t.i. hexanol), $m / z 101.097$ (t.i. hexanal) and $m / z$ 119.105; and of $m / z 79.06$ (t.i. benzene), $m / z 91.057$ (t.i. diethyl sulfide), $m / z 105.039$ (methional), $m / z 107.044$ (t.i. benzaldehyde), $m / z 107.088$ (t.i. ethyl benzene) and $m / z 125.059$ (t.i. benzyl alcohol).

Moreover, these volatilome results evidenced that all almond elite cultivars assessed in this study, except "Ferraduel" (\#44), were characterized by a less intense VOC profile than many of the Sicilian accessions. As for many other horticulture products, this lower VOC content might be the indirect consequence of a cultivar selection for the most oriented to the fruit productivity rather than to the quality ${ }^{9}$. Noticeably, it was possible to define several clusters of cultivars, among the Sicilian accessions, characterized by a considerable high content of compounds with a specific, and easy to be perceived, aroma note, like benzaldehyde (Fig. 1f) or phenyl ethyl alcohol (Fig. 1d). While benzaldehyde is the characteristic and predominant odour compound of bitter almond ${ }^{45}$, phenyl ethyl alcohol, associated with floral and rose aroma note, was already detected in several almond genotypes, but at low concentrations ${ }^{45,46}$. "Don Pitrino" (\#36), "Pizzuta grande" (\#80), "Comunista" (\#28), "Pilusedda" (\#76), "Mennula du nigliu" (\#57) and "Vaiana" (\#101) were some of the cultivars of our germplasm collection characterized by highest phenyl ethyl alcohol concentration. This feature might be interesting not only for the agro-food sector but also for the cosmetic industry ${ }^{47}$.

Taking into account the high genetic variability considered in this study, we aimed to uncover most of the possible VOC variability among Prunus dulcis genotypes. However, without a detailed sensory analysis, quantifying the relevance of each VOC might be too speculative, bearing also in mind the non-linear interaction of these molecules in determining consumer preference. For this reason, in order to reduce any possible statistical bias in the result interpretation, all data were analysed with unsupervised multivariate statistical methodologies (PCA and hierarchical clustering). Nonetheless, considering each quality trait independently (i.e. Supplementary Fig. 1) might be useful for a breeding approach aimed to introduce, or improve, a distinct quality trait to an elite breeding line.

To simplify the application of these results, we limited the number of VOC traits that have to be considered (Supplementary Fig. 3), according to the loading plots of the principal component analysis and to the results of previously published articles on almond aroma ${ }^{32,33,48-51}$. The content of each trait (including also some pomological feature such as fruit and kernel weights, kernel thickness, or flavour) was grouped based on the distribution quantile (low: 0-25\%; middle-low: 25-50\%; middle-high: 50-75\%; high: 75-100\%), calculated for both raw and roasted assessment (Supplementary Fig. 3). Accessions employed in the study can be consequently sorted and clustered according to the content of the trait of interest, which can be arbitrarily chosen as implemented in the dedicated webpage https://iuliiakhomenkofmach.shinyapps.io/QualySort $/{ }^{52}$.

\section{Definition of a robust SNP set and peach/almond synteny analysis}

The original set of 16,038 SNPs was filtered using the ASSIsT software ${ }^{53}$ resulting in the detection of 471 (2.9\%) robust polymorphic markers spanning the eight almond chromosomes. Among the discarded markers, 11,743 (73.2\%) were monomorphic, 2321 (14.5\%) failed in the amplification and the remaining 1503 (9.3\%) were characterized by the presence of null alleles. The relatively low number of failed SNPs confirmed the high synteny 
Pd. 1

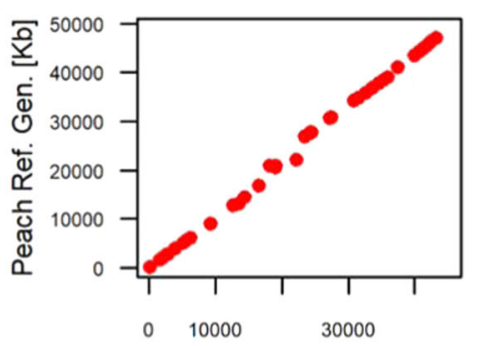

Almond Ref. Gen. [Kb]

Pd. 4

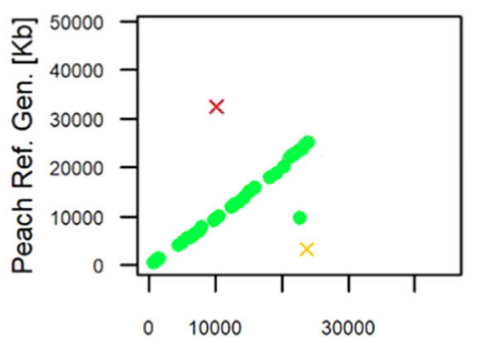

Almond Ref. Gen. [Kb]

Pd. 7

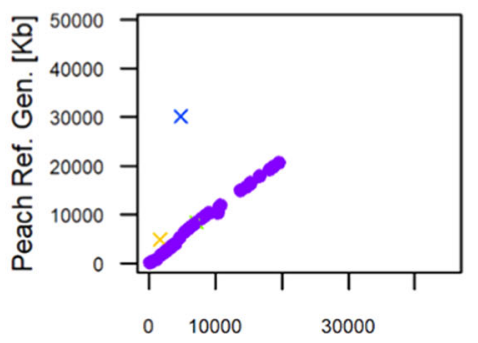

Almond Ref. Gen. [Kb]
Pd. 2

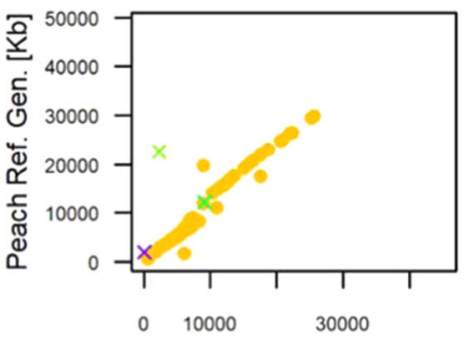

Almond Ref. Gen. [Kb]

Pd. 5

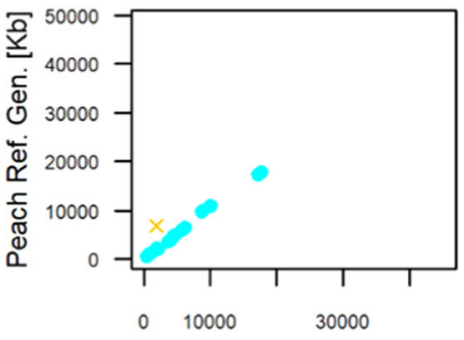

Almond Ref. Gen. [Kb]

Pd. 8

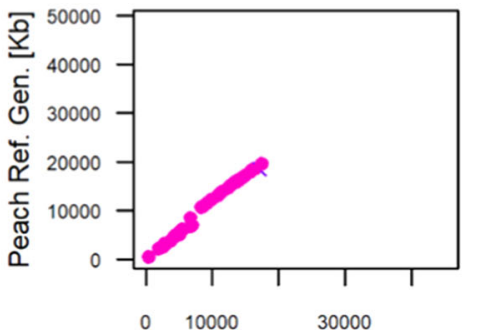

Almond Ref. Gen. [Kb]
Pd. 3

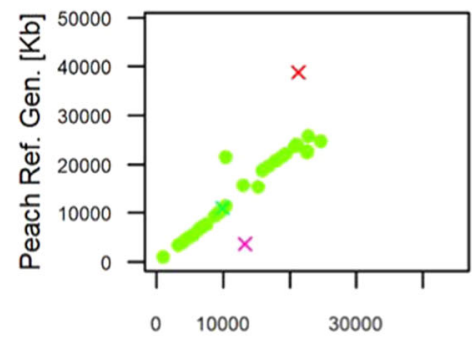

Almond Ref. Gen. [Kb]

Pd. 6

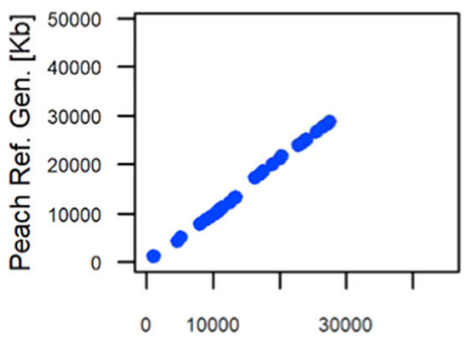

Almond Ref. Gen. [Kb]

Fig. 3 Collinearity plots between almond ( $\boldsymbol{x}$ axis) and peach ( $\boldsymbol{y}$ axis). Physical coordinates for SNP markers were retrieved from the Prunus dulcis Texas Genome v2.0 and the Prunus persica Whole-Genome Assembly v2.0 respectively. SNPs mapped in the same linkage groups in both species were represented as full dots while SNPs mapped in different linkage groups were represented as cross

between peach and almond genomes, nevertheless, the high fraction of monomorphic markers well reflected the fact that the probes were designed to target SNPs characterizing a different, although similar, species.

SNPs spanned over $199.7 \mathrm{Mb}$, covering most of the almond genome which is characterized by a genome size ranging from $227 \mathrm{Mb}$ (cultivar 'Texas') ${ }^{54}$ to $246 \mathrm{Mb}$ ('cultivar 'Laurenne') ${ }^{55}$. Pd1 was the longest linkage group (43.1 Mb) while the remaining ranged from $27.5 \mathrm{Mb}$ (Pd6) to $17.5 \mathrm{Mb}$ (Pd8) (Fig. 3, Supplementary Fig. 4).
The number of mapped SNPs per chromosome spanned from $22(\mathrm{Pd} 5)$ to $123(\mathrm{Pd} 2)$, with a mean value of 59, the average marker density was 1 marker every $424 \mathrm{~Kb}$ (Supplementary Table 3).

The physical position of the 471 SNPs on the almond ${ }^{54}$ and peach genome ${ }^{56}$ was highly consistent $\left(r^{2}=0.96\right)$ highlighting a high synteny and collinearity between the two species. 13 SNPs (2.7\%) mapped on different chromosomes in the two species. Pd1 and Pd6 did not show any inconsistencies and the SNP positions along the 
Table 2 Table summarizing the GWAS analysis for the fresh aromatic compounds characterizing almond kernels

\begin{tabular}{|c|c|c|c|c|}
\hline SNP & LG & Bp & $\begin{array}{l}\text { adjusted } \\
P \text { value }\end{array}$ & $\begin{array}{l}\text { Mass peak } \\
(\mathrm{m} / \mathrm{z})\end{array}$ \\
\hline Peach_AO_0868502 & $\mathrm{Pd} 8$ & 17442905 & 4.201470892 & 44.025 \\
\hline Peach_AO_0868502 & $\mathrm{Pd} 8$ & 17442905 & 4.071816888 & 45.033 \\
\hline SNP_IGA_269327 & $\mathrm{Pd} 2$ & 16486499 & 4.028810556 & 67.992 \\
\hline SNP_IGA_829830 & $\mathrm{Pd} 8$ & 5512121 & 4.157652959 & 73.064 \\
\hline SNP_IGA_574988 & $\mathrm{Pd} 5$ & 6207994 & 3.990959552 & 80.06 \\
\hline Peach_AO_0868502 & $\mathrm{Pd} 8$ & 17442905 & 4.075803665 & 81.041 \\
\hline SNP_IGA_269327 & $\operatorname{Pd} 2$ & 16486499 & 4.097908525 & 84.087 \\
\hline SNP_IGA_881173 & $\mathrm{Pd} 8$ & 17475606 & 5.503711552 & \\
\hline SNP_IGA_829830 & $\mathrm{Pd} 8$ & 5512121 & 4.14734958 & 85.067 \\
\hline SNP_IGA_269327 & $\mathrm{Pd} 2$ & 16486499 & 4.480595251 & 85.102 \\
\hline SNP_IGA_881173 & $\mathrm{Pd} 8$ & 17475606 & 4.988874658 & \\
\hline Peach_AO_0684073 & $\mathrm{Pd} 6$ & 26559511 & 4.288725249 & 87.081 \\
\hline Peach_AO_0868502 & $\mathrm{Pd} 8$ & 17442905 & 4.50974781 & 89.061 \\
\hline Peach_AO_0423401 & $\mathrm{Pd} 4$ & 4607767 & 4.416783526 & 97.066 \\
\hline SNP_IGA_881173 & $\mathrm{Pd} 8$ & 17475606 & 6.013452832 & \\
\hline Peach_AO_0423401 & $\mathrm{Pd} 4$ & 4607767 & 4.039290176 & 99.082 \\
\hline SNP_IGA_353861 & $\mathrm{Pd} 3$ & 18886145 & 6.774104184 & 99.117 \\
\hline SNP_IGA_269327 & $\mathrm{Pd} 2$ & 16486499 & 4.080811033 & 101.097 \\
\hline SNP_IGA_881173 & $\mathrm{Pd} 8$ & 17475606 & 5.125383663 & \\
\hline SNP_IGA_269327 & $\mathrm{Pd} 2$ & 16486499 & 4.033428856 & 103.115 \\
\hline SNP_IGA_881173 & $\mathrm{Pd} 8$ & 17475606 & 4.71831783 & \\
\hline SNP_IGA_269327 & $\mathrm{Pd} 2$ & 16486499 & 4.626074734 & 109.103 \\
\hline SNP_IGA_881173 & $\mathrm{Pd} 8$ & 17475606 & 8.525897285 & 111.118 \\
\hline SNP_IGA_881173 & $\mathrm{Pd} 8$ & 17475606 & 4.109750783 & 113.099 \\
\hline Peach_AO_0070614 & $\mathrm{Pd} 1$ & 22157470 & 4.988201194 & 115.114 \\
\hline SNP_IGA_269327 & $\mathrm{Pd} 2$ & 16486499 & 4.139158493 & 119.106 \\
\hline SNP_IGA_881173 & $\mathrm{Pd} 8$ & 17475606 & 5.081147321 & \\
\hline SNP_IGA_269327 & $\mathrm{Pd} 2$ & 16486499 & 4.101227804 & 121.12 \\
\hline SNP_IGA_881173 & $\mathrm{Pd} 8$ & 17475606 & 4.561356555 & \\
\hline SNP_IGA_269327 & $\mathrm{Pd} 2$ & 16486499 & 4.830909992 & 127.114 \\
\hline SNP_IGA_881173 & $\mathrm{Pd} 8$ & 17475606 & 5.85241464 & \\
\hline SNP_IGA_881173 & $\mathrm{Pd} 8$ & 17475606 & 5.650769836 & 129.129 \\
\hline Peach_AO_0868502 & $\mathrm{Pd} 8$ & 17442905 & 4.0565555312 & 134.973 \\
\hline SNP_IGA_881173 & $\mathrm{Pd} 8$ & 17475606 & 5.979463513 & 143.11 \\
\hline SNP_IGA_353861 & $\mathrm{Pd} 3$ & 18886145 & 4.55573974 & 143.145 \\
\hline SNP_IGA_881173 & $\mathrm{Pd} 8$ & 17475606 & 6.358830393 & 147.137 \\
\hline SNP_IGA_881173 & $\mathrm{Pd} 8$ & 17475606 & 4.053380905 & 157.161 \\
\hline SNP_IGA_182843 & $\mathrm{Pd} 2$ & 5229709 & 4.034321274 & 159.139 \\
\hline SNP_IGA_353861 & $\mathrm{Pd} 3$ & 18886145 & 7.72892178 & \\
\hline
\end{tabular}

For each SNP exceeding the GWAS significance threshold, the corresponding physical position according to the Prunus dulcis Texas Genome v2.0 was reported together with the relative FDR-adjusted $p$ value (expressed as $-\log 10$ $p$ value) and the corresponding volatile organic compounds (VOC) mass peak

almond and peach linkage groups showed an $r^{2}=0.997$ (Pd1) and 0.998 (Pd6), (Fig. 3). The other linkage groups were characterized by the occurrence of $1-3 \mathrm{SNP}(\mathrm{s})$ mapped in different linkage groups in the two species (Fig. 3). The high synteny between peach and almond was in agreement with previous studies highlighting that most of the genomes of the Prunus species can be considered as a single entity ${ }^{19,54,57}$.

\section{Analysis of genetic structure}

The level of genetic stratification was assessed using the Bayesian approach implemented in the software STRUCTURE $^{58}$. Among the different number of subpopulations postulated, $K=3$ showed the highest likelihood ( $\Delta K=346$ ) followed by $K=7$ and $K=2$ showing similar likelihoods $(\Delta K=151$ and 116 respectively, Supplementary Fig. 5).

Figure 4A showed the genetic configuration of the 106 individuals for $K=3 ; 45$ accessions were characterized by a clear predominance $(\mathrm{Q} i \geq 0.8)$ of one of the three subpopulations, in particular: 19 accessions were predominantly characterized by Subpop 1 while both Subpop2 and Subpop3 were represented by 13 accessions each. The remaining 61 genotypes showed a higher level of admixture (Supplementary Table 4). The SNP data analysis and the structure results confirmed the origin of the self-compatible cultivar "Supernova" (\#93) as a mutant of the self-incompatible "Tuono" $(\# 98)^{59}$ with the two cultivars characterized by an identical genotypic profile for all the SNP tested (and consequently an identical genetic structure for all the Ks postulated, Supplementary Table 4, Supplementary Fig. 6). Overall, the Apulian and International accessions were characterized by a similar contribution of Subpop1 (12.3\% and $12.1 \%$ respectively); then the most represented subpopulations were Subpop2 for the International group (54.3\%) and Subpop3 (46.1\%) for the Apulian accessions (Fig. 4b). Conversely, the Sicilian accessions were characterized by a much higher contribution of Subpop1 (44.8\%) while Subpop2 and Subpop3 (31.1\% and $24.1 \%$ respectively) were less represented compared to the Apulian and International accessions (Fig. 4b). The widely cultivated Sicilian accessions "Pizzuta d'Avola" (\#78) and "Fascionello" (\#39), both characterized by a high prevalence of Subpop1 ( $\mathrm{Q} i=0.99$ and 0.98 respectively), were genetically distant from the Apulian and International cultivars (Fig. 4a, Supplementary Table 4), in agreement with previous genetic population studies based on $\mathrm{SSRs}^{29}$.

\section{Identification of genomic regions underlying VOCs production}

Marker-trait association approaches were successfully employed in most of the tree crops to identify molecular markers in strong LD with the causative gene(s) influencing a trait of agronomical interest. In this study, molecular and phenotypic data were employed for a preliminary application of a GWAS analysis to identify molecular markers linked to the VOC production of the fresh and roasted almond kernel.

Among the 150 mass peaks related to the VOC profile of fresh almond, 31 were characterized by significant markertrait associations for at least one of the SNP tested. Although with different relative frequencies, significant SNPs were 
Table 3 Table summarizing the GWAS analysis for the roasted aromatic compounds characterizing almond kernels

\begin{tabular}{|c|c|c|c|c|}
\hline SNP & LG & $\mathrm{Bp}$ & $\begin{array}{l}\text { adjusted } \\
P \text { value }\end{array}$ & $\begin{array}{l}\text { Mass peak } \\
(\mathrm{m} / \mathrm{z})\end{array}$ \\
\hline Peach_AO_0260252 & $\mathrm{Pd} 2$ & 11738618 & 5.480565119 & 34.996 \\
\hline Peach_AO_0267535 & $\mathrm{Pd} 2$ & 12792357 & 5.445052149 & \\
\hline SNP_IGA_811258 & $\mathrm{Pd} 3$ & 13261568 & 5.549766949 & 43.018 \\
\hline SNP_IGA_811258 & $\mathrm{Pd} 3$ & 13261568 & 4.240584024 & 44.025 \\
\hline SNP_IGA_811258 & $\mathrm{Pd} 3$ & 13261568 & 4.523506694 & 61.028 \\
\hline Peach_AO_0280324 & $\mathrm{Pd} 2$ & 8937322 & 5.55589741 & 63.029 \\
\hline SNP_IGA_829830 & $\mathrm{Pd} 8$ & 5512121 & 4.241860664 & 73.064 \\
\hline SNP_IGA_829830 & $\mathrm{Pd} 8$ & 5512121 & 4.269403089 & 75.072 \\
\hline Peach_AO_0539745 & $\operatorname{Pd} 5$ & 1973367 & 4.2981529 & 83.076 \\
\hline SNP_IGA_881173 & $\mathrm{Pd} 8$ & 17475606 & 4.330316917 & 83.087 \\
\hline SNP_IGA_269327 & $\mathrm{Pd} 2$ & 16486499 & 4.152037221 & 85.102 \\
\hline SNP_IGA_881173 & $\mathrm{Pd} 8$ & 17475606 & 4.918056605 & \\
\hline SNP_IGA_829830 & $\mathrm{Pd} 8$ & 5512121 & 4.441318507 & 91.075 \\
\hline Peach_AO_0539745 & $\mathrm{Pd} 5$ & 1973367 & 4.206372997 & 93.091 \\
\hline SNP_IGA_881173 & $\mathrm{Pd} 8$ & 17475606 & 5.016508288 & 97.102 \\
\hline SNP_IGA_353861 & $\mathrm{Pd} 3$ & 18886145 & 7.040643097 & 99.117 \\
\hline SNP_IGA_881173 & $\mathrm{Pd} 8$ & 17475606 & 4.298774607 & 101.097 \\
\hline Peach_AO_0238372 & $\mathrm{Pd} 2$ & 10989084 & 4.31862644 & 103.052 \\
\hline SNP_IGA_829830 & $\mathrm{Pd} 8$ & 5512121 & 4.352859143 & \\
\hline SNP_IGA_881173 & $\mathrm{Pd} 8$ & 17475606 & 4.484961281 & 103.115 \\
\hline SNP_IGA_829830 & $\mathrm{Pd} 8$ & 5512121 & 4.288455871 & 105.09 \\
\hline SNP_IGA_574988 & $\mathrm{Pd} 5$ & 6207994 & 4.359176154 & 107.044 \\
\hline SNP_IGA_269327 & $\mathrm{Pd} 2$ & 16486499 & 4.511247633 & 109.103 \\
\hline SNP_IGA_881173 & $\mathrm{Pd} 8$ & 17475606 & 4.867016526 & \\
\hline Peach_AO_0047516 & Pd 1 & 14445732 & 4.776994667 & 111.118 \\
\hline SNP_IGA_881173 & $\mathrm{Pd} 8$ & 17475606 & 5.582937641 & \\
\hline SNP_IGA_881173 & $\mathrm{Pd} 8$ & 17475606 & 4.671631163 & 113.099 \\
\hline Peach_AO_0070614 & Pd 1 & 22157470 & 4.010715179 & 115.114 \\
\hline SNP_IGA_829830 & $\mathrm{Pd} 8$ & 5512121 & 4.172855296 & 121.067 \\
\hline SNP_IGA_563930 & $\mathrm{Pd} 5$ & 4387202 & 4.000740594 & 125.134 \\
\hline SNP_IGA_881173 & $\mathrm{Pd} 8$ & 17475606 & 5.591961603 & \\
\hline SNP_IGA_269327 & $\mathrm{Pd} 2$ & 16486499 & 4.139867267 & 127.114 \\
\hline SNP_IGA_881173 & $\mathrm{Pd} 8$ & 17475606 & 4.667857362 & \\
\hline Peach_AO_0814869 & $\mathrm{Pd} 8$ & 5093115 & 4.148932031 & 127.148 \\
\hline SNP_IGA_881173 & $\mathrm{Pd} 8$ & 17475606 & 5.254941658 & 129.129 \\
\hline SNP_IGA_881173 & $\mathrm{Pd} 8$ & 17475606 & 4.346475813 & 143.11 \\
\hline SNP_IGA_881173 & $\mathrm{Pd} 8$ & 17475606 & 4.205182451 & 147.137 \\
\hline
\end{tabular}

Table 3 continued

\begin{tabular}{llrll}
\hline SNP & LG & Bp & \multicolumn{1}{l}{$\begin{array}{l}\text { adjusted } \\
\boldsymbol{P} \text { value }\end{array}$} & $\begin{array}{l}\text { Mass peak } \\
(\mathbf{m} / \mathbf{z})\end{array}$ \\
\hline Peach_AO_0423401 & Pd 4 & 4607767 & 4.183997572 & 155.178 \\
SNP_IGA_881173 & Pd 8 & 17475606 & 4.404726319 & 157.161 \\
SNP_IGA_353861 & Pd 3 & 18886145 & 4.229546071 & 159.139 \\
\hline
\end{tabular}

For each SNP exceeding the GWAS significance threshold, the corresponding physical position according to the Prunus dulcis Texas Genome v2.0 was reported together with the relative FDR-adjusted $p$ value (expressed as $-\log 10$ $p$ value) and the corresponding volatile organic compounds (VOC) mass peak

detected in all linkage groups except Pd7. Pd8 resulted significantly associated with 21 VOC mass peaks while for the other linkage groups, the number of VOC mass peaks exceeding the significance threshold ranged from 1 (Pd1, Pd5 and Pd6) to 10 (Pd2) (Table 2). As for the VOCs contributing the roasted volatile profile of almonds, 33 mass peaks showed significant association (Table 3). Similarly, to what registered for the VOC profiling of fresh almonds, the highest number of signals were detected in Pd8 (21) while no significant associations were observed for Pd6 and Pd7 (Table 3).

Among the VOC mass peaks showing a significant association, 15 were in common between fresh and roasted almond kernels (Tables 2 and 3). All those mass peaks were mapped in the same genetic regions in both VOC assessments except for $\mathrm{m} / \mathrm{z} 44.025$ (unknown molecule, mapped in Pd8 and in Pd3 respectively, Tables 2 and 3). Among the 15 VOCs detected in both fresh and roasted treatments, $m / z$ 73.064, $\mathrm{m} / \mathrm{z}$ 85.102, $\mathrm{m} / \mathrm{z}$ 99.117, $\mathrm{m} / \mathrm{z} 103.115, \mathrm{~m} / \mathrm{z} 111.118$, $\mathrm{m} / \mathrm{z}$ 113.099, $\mathrm{m} / \mathrm{z}$ 127.114, $\mathrm{m} / \mathrm{z} \quad 143.11$ and $\mathrm{m} / \mathrm{z}$ 157.161 showed significant differences between the two treatments (Table 1), suggesting that, even if the quantity of the VOC changed significantly during roasting, the genetic region associated to the trait remained the same.

In both fresh and roasted phenotypes, the significant signals in $\mathrm{Pd} 8$ were detected in two genetic regions: at around $5.5 \mathrm{Mb}$ (2 and 7 SNPs respectively for fresh and roasted kernels) and 17.4 Mb (19 and 14 SNPs respectively) suggesting the existence of either a cluster of genes underlying the synthesis of different aromatic compounds or the presence of common genetic regulation systems (Fig. 5). Further study with higher marker density will help to clarify the number and function of the genes located in Pd8.).

\section{Analysis of LD}

The analysis of the non-random association between loci through a whole-genome LD decay scan provides insights on the population genetic forces structuring the germplasm collection in the analysis. The mean $r^{2}$ for all intrachromosomal loci pairs was equal to 0.083 , while the chromosome-wise LD ranged from $0.076(\mathrm{Pd} 2)$ to 
A

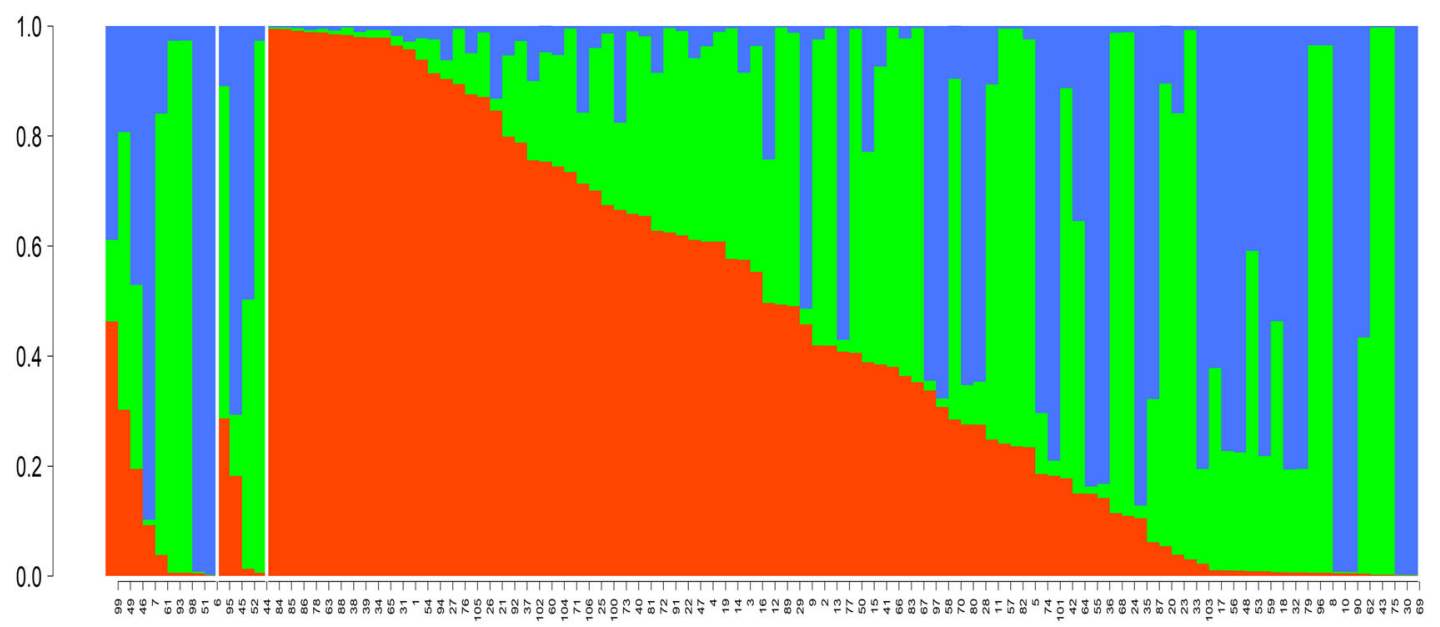

B
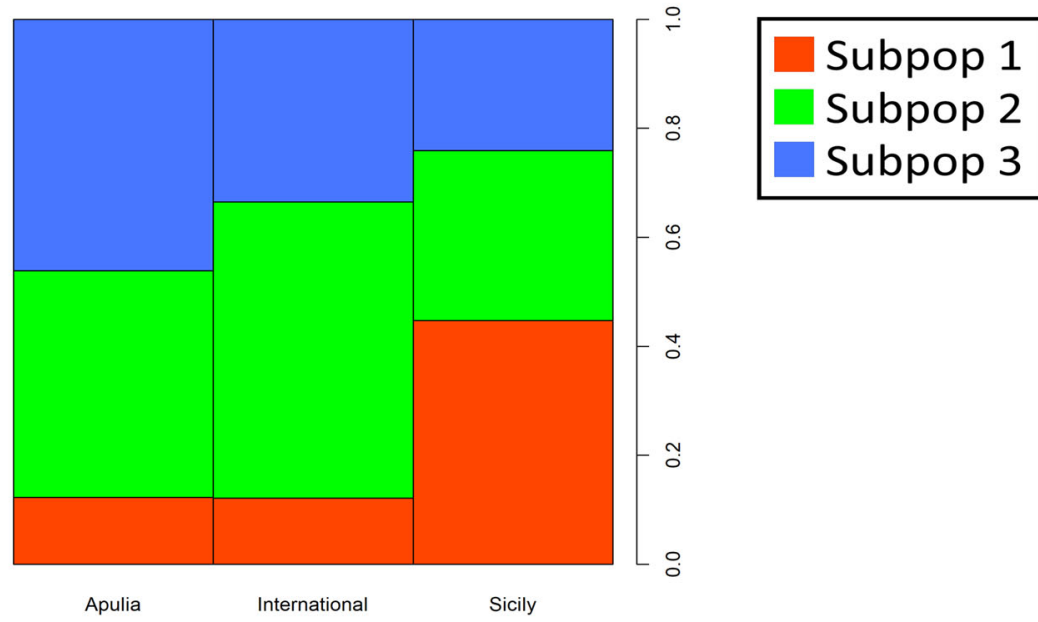

Fig. 4 Structure plots for $\boldsymbol{K}=\mathbf{3}$. The three subpopulations postulated were coloured in red (Subpop1), in green (Subpop2) and blue (Subpop3) as specified in the legend. A Individual results of the Structure analysis: the 106 accessions were ordered from left to right according to the different origin: Apulian (9), International (4), Sicilian (93), each group is separated by a white vertical line. B Contribution of the three subpopulations according to the different origin of the accessions

0.181 (Pd5). The LD decay detected in this study was slightly higher than in previous reports (mean $r^{2}=0.04^{23}$ ). The highly significant $r^{2}$ threshold, calculated as the 95th percentile of the $r^{2}$ distribution, was 0.41 , corresponding to LD blocks of $\sim 60 \mathrm{~Kb}$ (Fig. 6). LD in almond decayed faster than peach ${ }^{60-63}$, apricot ${ }^{64}$ and cherry ${ }^{65}$. Among the different causes taking part in the species-specific LD decay, the mating system (out-crossing versus self-compatible) is probably the most important factor influencing the different LD decays in Prunus. While almond and cherry, with some rare exceptions, are out-crossing species, peach and apricot are self-compatible and the subsequent self-pollination results in lower heterozygosity and slower LD decay ${ }^{66}$.

\section{Conclusion}

Future breeding programs, focused on the optimization of consumer perceived quality, need to consider almond VOC modification related to genetic variability, environmental effect, and transformation. This can be achieved only with a more objective and precise identification of the best performing cultivars to be used as superior parental lines in combination with a reliable phenotyping methodology and genotyping assay. This investigation supports the use of PTR-ToF-MS as an accurate and objective phenotyping tool to evaluate the VOC profile of almonds. Indeed, most of the molecules that were previously identified on a limited number of almond accessions by gas chromatographic 


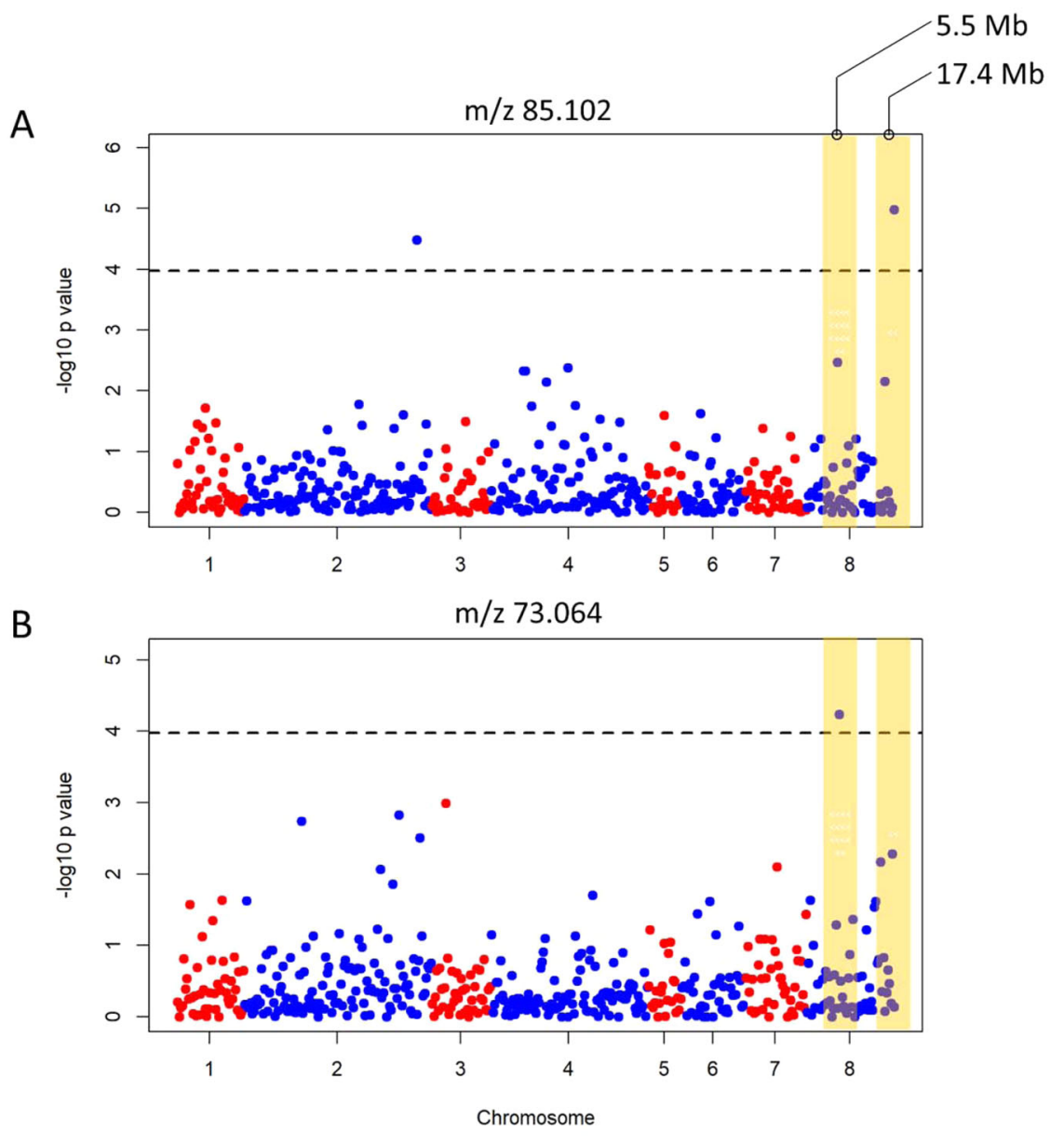

Fig. 5 Manhattan plots illustrating the significant marker-trait association between genetic data and two mass peaks: $\mathrm{m} / \mathbf{z} 85.102$ (fresh assessment) and $m / z 73.064$ (roasted assessment). The two genomic regions in $\mathrm{Pd} 8$ in which the significant signals were detected were highlighted in yellow

analysis $^{33,35,36}$ were detected in this broad germplasm collection.

A preliminary GWAS analysis enabled the identification of $63 \mathrm{VOC}$ mass peaks (related to fresh and/or roasted treatment) showing a significant phenotype-genotype association. The detection of molecular markers in close linkage to several aroma components could be of great interest for the set-up of marker-assisted selection (MAS) approaches in novel breeding schemes to enhance the almond aroma. However, a better understanding of genes and enzymes involved in the VOC production, during kernel ripening or during roasting, is still needed. Further studies aimed at a real-time VOC assessment during almond roasting will provide a more complete overview of the volatilome of almond kernel, while the availability of a dedicated almond SNP array will allow a better genetic resolution for the detection of candidate genes regulating the aromatic characteristics of almond.

\section{Materials and methods Plant materials}

The germplasm was composed by 106 almond accessions maintained in the ex situ germplasm collection held at the 'Museo vivente del mandorlo Francesco Monastra', located in Sicily (latitude: 37.2921094, longitude: 13.5817574 , altitude: $121 \mathrm{~m}$ above sea level). The germplasm collection was mainly composed of Sicilian almond accessions selected through centuries for their agronomic traits of interest (e.g. fruit quality, resistance to biotic or abiotic stress, shell hardiness) complemented with widely known national and international cultivars as outlined in Supplementary Table 5. For each accession, almond kernels were harvested from four plants, grown under standard agronomical practices. Data related to the pomological characteristics of the fruit and kernel were retrieved from ref. ${ }^{67}$. 


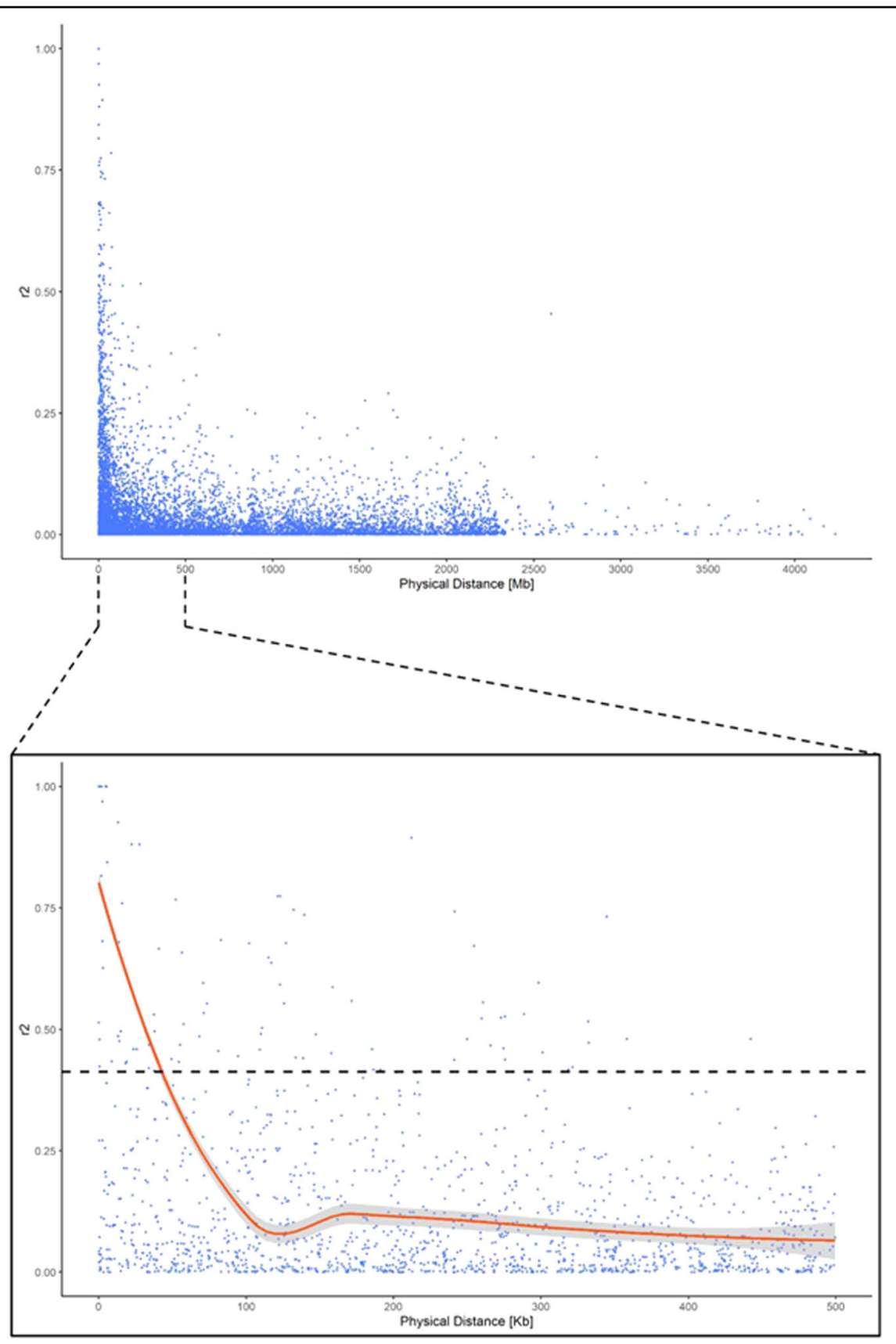

Fig. 6 Genome-wide scatterplot of linkage disequilibrium decay $\left(r^{2}, y\right.$ axis) against the genetic distance (Mb, $x$ axis) for pairs of linked SNPs across the eight linkage groups. In the window below, only the first $500 \mathrm{Mb}$ were displayed together with a LOESS fitting curve summarizing the linkage disequilibrium decay at increasing physical distances (red continuous line) and the relative confidence interval (grey area). The intersection between the LOESS fitting curve and the 95th percentile of the $r^{2}$ distribution (black dashed line) was taken as the threshold value to consider two markers in close linkage disequilibrium

VOC analysis by proton transfer-time of flight-mass spectrometer

Almond kernels were collected at full ripening stage according to the maturity period of the different accessions (from mid-August to mid-September 2019) and conserved at $4{ }^{\circ} \mathrm{C}$ prior to the analysis. Three biological replicates of $3 \mathrm{~g}$ of sliced sample, each obtained by five fresh unpeeled almond kernels, were inserted into $20 \mathrm{~mL}$ glass vials equipped with PTFE/silicone septa (Agilent, Cernusco sul Naviglio, Italy). Measurements of almond VOCs were 
performed on three biological replicates with a commercial PTR-ToF-MS 8000 (Ionicon Analytik GmbH, Innsbruck, Austria ${ }^{12}$ ). The drift tube conditions were as follows: $110^{\circ} \mathrm{C}$ drift tube temperature, 2.8 mbar drift pressure, $428 \mathrm{~V}$ drift voltage, ion funnel $(18 \mathrm{~V})$. This leads to an $\mathrm{E} / \mathrm{N}$ ratio of about 130 Townsend (Td), with $\mathrm{E}$ corresponding to the electric field strength and $\mathrm{N}$ to the gas number density $\left(1 \mathrm{Td}=10^{-17} \mathrm{Vcm}^{-2}\right)$. The sampling time per channel of ToF acquisition was $0.1 \mathrm{~ns}$, amounting to 350,000 channels for a mass spectrum ranging up to $m / z=400$. The sample headspace was withdrawn through PTR-MS inlet with $40 \mathrm{sccm}$ flow for 60 cycles resulting in an analysis time of $60 \mathrm{~s} / \mathrm{sample}$. Pure nitrogen was flushed continuously through the vial to prevent pressure drop. Each measurement was conducted automatically after $25 \mathrm{~min}$ of sample incubation at $40^{\circ} \mathrm{C}$ and $5 \mathrm{~min}$ between each measurement was applied in order to prevent memory effect. All steps of measurements were automated by an adapted GC autosampler (MPS Multipurpose Sampler, GERSTEL) coupled to PTR-ToF-MS. After the PTR-ToF-MS measurement of fresh almonds, each vial, without cup, was transferred into an oven (WTB Binder, Germany) at $150^{\circ} \mathrm{C}$ for $15 \mathrm{~min}$ to achieve a medium roast. These roasting conditions were decided based on literature information ${ }^{35,36}$ and on preliminary tests performed on almond kernel genotypes, characterized by different shapes and sizes profile of roasted almonds was assessed in the same way of fresh samples.

The analysis of PTR-ToF-MS spectra proceeded as described in Farneti et al. ${ }^{11}$. The array of masses detected with PTR-ToF-MS was reduced by applying noise and correlation coefficient thresholds. The first removed peaks that were not significantly different from blank samples; the latter excluded peaks with over $99 \%$ correlation, which mostly correspond to isotopes of monoisotopic masses ${ }^{11}$.

R.4.0.2 ${ }^{68}$ internal statistical functions and the external packages "mixOmics", "heatmap3", "dendextend", and "ggplot2" were used for the multivariate statistical methods (PCA, heatmap, hierarchical clustering, and tanglegram) and for the "Lollipop graph" employed in this work $^{69-72}$.

\section{SNP Genotyping and synteny analysis}

Total DNA was extracted from fresh leaf tissue using the CTAB extraction method proposed by Doyle and Doyle $^{73}$ following the protocol described by Distefano and colleagues $^{29}$. The almond germplasm collection was genotyped employing the Illumina Infinium ${ }^{\circledR} 18 \mathrm{~K}$ Peach SNP array ${ }^{31}$. The use of an SNP array developed for peach is due both to the lack of SNP arrays specifically designed for almond and the high marker transferability between the two species ${ }^{57}$. Robust SNPs were filtered using the ASSIsT software ${ }^{53}$ with default parameters (allowed missing data $=0.05$, unexpected genotype threshold $=$ 0.003 , frequency rare allele $=0.05$ ). Markers were ordered along the eight linkage groups using the Prunus dulcis Texas Genome v2.0 ${ }^{54}$, while the Prunus persica WholeGenome Assembly v2.0 ${ }^{56}$ was employed for collinearity analysis.

\section{Deciphering the population structure of the almond collection}

The most probable number of subpopulations $(K)$ characterizing the 106 accessions was assessed using the STRUCTURE software v2.3.4 ${ }^{58}$. The $K$ tested ranged from 1 to 10 . For each $K$, five independent runs were carried out with a burn-in period of 10,000 and 100,000 Markov chain Monte Carlo replications after burn-in. The $K$ value that best fits the data was assessed by calculating the DeltaK value $^{74}$ as implemented in the STRUCTURE HARVESTER program ${ }^{75}$ (http://taylor0.biology.ucla.edu/ structureHarvester/). The five independent runs were integrated using the CLUMPP software ${ }^{76}$ and resulting $Q$ matrices were displayed in $R^{68}$. Accessions showing a membership coefficient $\left(Q_{\mathrm{i}}\right)$ equal or higher than 0.8 were assigned to a subpopulation, while the others were classified as 'admixed"77.

\section{Phenotype-genotype association analysis}

Phenotypic and genotypic data were integrated in a GWAS analysis using the Efficient Mixed-Model Association eXpedited (EMMAX) implemented in the 'GWAS' function of the rrBLUP R package ${ }^{78}$. The GWAS model employed in the analysis is expressed as follows:

$$
y=X \beta+Z g+S \tau+\varepsilon
$$

where $\beta$ is a vector of fixed effects modelling both environmental factors and population structure, the variables $g$ and $\tau$ models the genetic background of each line as a random effect and the additive SNP effect as a fixed effect respectively; $\varepsilon$ summarizes the residual variance. The GWAS model employed takes genetic structure and kinship matrix as covariates to correct for genetic stratification and parental relationship. To minimize typeone errors, significant associations were detected after correcting the $p$ value for multiple testing using the false discovery rate (FDR) method ${ }^{79}$. FDR is computed using the $q$ value package in $\mathrm{R}^{80}$; SNPs exceeding the FDR threshold rate of 0.05 (represented by a dashed line in the Manhattan plot) were considered significantly associated with the phenotype.

\section{LD, QTL anchoring and in silico gene annotation}

The LD decay was calculated using the $\mathrm{R}$ package sommer v2.9 ${ }^{81}$. The genome-wide LD decay was visualized using the R software ${ }^{68}$ plotting the LD parameter $r^{2}$ against the corresponding physical distance. The 95th percentile of the $r^{2}$ distribution was taken as the threshold 
value to consider two markers in close $\mathrm{LD}^{82}$. The $r^{2}$ threshold baseline was matched to the locally weighted polynomial regression-based fitting curve (LOESS) to estimate the average LD decay distance using the 'stats' $R$ package $^{68}$.

\section{Acknowledgements}

The work was carried out in the frame of the PON "AIM: Attrazione e Mobilità Internazionale", project number $1848200-2$. The work was partially founded by the project entitled: 'Strategie di innovazione sostenibile per la gestione conservativa del suolo, la salvaguardia dell'agrobiodiversità e la valutazione quanti-qualitativa delle produzioni meditteranee' supported by the University of Catania. We would also like to thank Simone Larger for performing the genotyping.

\section{Author details}

'Department of Agriculture, Food and Environment (Di3A), University of Catania, via Valdisavoia 5, 95123 Catania, Italy. ${ }^{2}$ Research and Innovation Centre, Fondazione Edmund Mach, San Michele all' Adige, Trento, Italy. ${ }^{3}$ Dipartimento di Architettura, University of Palermo, Viale delle Scienze, Ed. 14 90128, Palermo, Italy. ${ }^{4}$ National Center for Citrus Improvement, College of Horticulture and Landscape, Hunan Agricultural University, Changsha, China

\section{Author contributions}

M.D.G. and G.D. conceived the study. F.S. designed the plant population. B.F. I.K., G.M., L.B. and M.T. generated the phenotypic and genotypic data. M.D.G. B.F., I.K. and A.M. performed the analysis with the support of A.G., S.L.M. and F.B. for suggestions and interpretations. M.D.G., B.F., G.D. and I.K. wrote the manuscript. All authors revised and approved the final version of the manuscript.

\section{Conflict of interest}

The authors declare that they have no conflict of interest.

Supplementary Information accompanies this paper at (https://doi.org/ 10.1038/s41438-021-00465-7).

Received: 4 September 2020 Revised: 11 December 2020 Accepted: 17 December 2020

Published online: 01 February 2021

\section{References}

1. FAOstat. Agriculture Data. 2018

2. Zohary, D. \& Hopf, M. Domestication of plants in the Old World: the origin and spread of cultivated plants in West Asia, Europe and the Nile Valley. (Oxford University Press, Oxford, UK, 2000).

3. Klein, A. M. et al. Wild pollination services to California almond rely on seminatural habitat. J. Appl. Ecol. 49, 723-732 (2012).

4. Kodad, O. et al. Oil content, fatty acid composition and tocopherol concentration in the Spanish almond genebank collection. Sci. Hortic. 177, 99-107 (2014).

5. Currò, S. et al. Analysis of S-allele genetic diversity in sicilian almond germplasm comparing different molecular methods. Plant Breed. 134, 713-718 (2015).

6. Tamura, M. et al. Identification of self-incompatibility genotypes of almond by allele-specific PCR analysis. Theor. Appl. Genet. 101, 344-349 (2000).

7. Channuntapipat, C. et al. Identification of incompatibility genotypes in almond (Prunus dulcis Mill.) using specific primers based on the introns of the S-alleles. Plant Breed. 122, 164-168 (2003)

8. El Hadi, M. A. M., Zhang, F. J., Wu, F. F., Zhou, C. H. \& Tao, J. Advances in fruit aroma volatile research. Molecules 18, 8200-8229 (2013).

9. Klee, H. J. Improving the flavor of fresh fruits: genomics, biochemistry, and biotechnology. N. Phytol. 187, 44-56 (2010)

10. Klee, H. J. \& Tieman, D. M. The genetics of fruit flavour preferences. Nat. Rev. Genet. 19, 347-356 (2018).

11. Farneti, B. et al. Exploring blueberry aroma complexity by chromatographic and direct-injection spectrometric techniques. Front. Plant Sci. 8, 1-19 (2017).
12. Farneti, B. et al. Genome-wide association study unravels the genetic control of the apple volatilome and its interplay with fruit texture. J. Exp. Bot. $\mathbf{6 8}$, 1467-1478 (2017).

13. Acierno, V., Fasciani, G., Kiani, S., Caligiani, A. \& van Ruth, S. PTR-QiToF-MS and $\mathrm{HSI}$ for the characterization of fermented cocoa beans from different origins. Food Chem. 289, 591-602 (2019).

14. Yener, $\mathrm{S}$. et al. Monitoring single coffee bean roasting by direct volatile compound analysis with proton transfer reaction time-of-flight mass spectrometry. J. Mass Spectrom. 51, 690-697 (2016).

15. Carbone, F. et al. Development of molecular and biochemical tools to investigate fruit quality traits in strawberny elite genotypes. Mol. Breed. 18, 127-142 (2006).

16. Costa, F. et al. QTL validation and stability for volatile organic compounds NOCs) in apple. Plant Sci. 211, 1-7 (2013).

17. Zargar, S. M. et al. Recent advances in molecular marker techniques: Insight into QTL mapping, GWAS and genomic selection in plants. J. Crop Sci. Biotechnol. 18, 293-308 (2015).

18. Rasheed, A. et al. Crop breeding chips and genotyping platforms: progress, challenges, and perspectives. Mol. Plant 10, 1047-1064 (2017).

19. Aranzana, M. J. et al. Prunus genetics and applications after de novo genome sequencing: achievements and prospects. Hortic. Res. https://doi.org/10.1038/ s41438-019-0140-8 (2019).

20. Sánchez-Pérez, R., Howad, W., Dicenta, F., Arús, P. \& Martínez-Gómez, P. Mapping major genes and quantitative trait loci controlling agronomic traits in almond. Plant Breed. 126, 310-318 (2007).

21. Font i Forcada CF, i Martí ÀF, I Company RS. Mapping quantitative trait loci for kernel composition in almond. BMC Genet. https://doi.org/10.1186/1471-215613-47 (2012).

22. Fernández i Martí, A., Font i Forcada, C. \& Socias i Company, R. Genetic analysis for physical nut traits in almond. Tree Genet. Genomes 9, 455-465 (2013).

23. Font i Forcada, C., Velasco, L., Socias i Company, R. \& Fernández i Martí, Á. Association mapping for kernel phytosterol content in almond. Front. Plant Sci. 6. 1-12 (2015).

24. Cantin, C. M., Wang, X. W., Almira, M., Arús, P. \& Eduardo, I. Inheritance and QTL analysis of chilling and heat requirements for flowering in an interspecific almond $x$ peach (Texas $x$ Earlygold) F2 population. Euphytica https://doi.org/ 10.1007/s10681-020-02588-9 (2020)

25. Baró-Montel, N. et al. Exploring sources of resistance to brown rot in an interspecific almond $\times$ peach population. J. Sci. Food Agric. 99, 4105-4113 (2019).

26. Fresnedo-Ramírez, J. et al. QTL mapping of pomological traits in peach and related species breeding germplasm. Mol. Breed. 35, 166 (2015).

27. Rafalski, J. A. Association genetics in crop improvement. Curr. Opin. Plant Biol. 13, 174-180 (2010).

28. Mackay, I. \& Powell, W. Methods for linkage disequilibrium mapping in crops. Trends Plant Sci. 12, 57-63 (2007).

29. Distefano, G. et al. Genetic diversity and relationships among Italian and foreign almond germplasm as revealed by microsatellite markers. Sci. Hortic. 162, 305-312 (2013).

30. Distefano, G. et al. HRM analysis of chloroplast and mitochondrial DNA revealed additional genetic variability in Prunus. Sci. Hortic. 197, 124-129 (2015).

31. Gasic, K. et al. Development and Evaluation of a 9 K SNP Addition to the Peach Ipsc 9 K SNP Array v1. In: American Society for Horticultural Science, annual conference (2019).

32. Xiao, L. et al. HS-SPME GC/MS characterization of volatiles in raw and dryroasted almonds (Prunus dulcis). Food Chem. 151, 31-39 (2014).

33. Erten, E. S. \& Cadwallader, K. R. Identification of predominant aroma components of raw, dry roasted and oil roasted almonds. Food Chem. 217, 244-253 (2017).

34. Bernal, J., Manzano, P., Diego, J. C., Bernal, J. L. \& Nozal, M. J. Comprehensive two-dimensional gas chromatography coupled with static headspace sampling to analyze volatile compounds: application to almonds. J. Sep. Sci. 37, 675-683 (2014).

35. Franklin, L. M. et al. Chemical and sensory characterization of oxidative changes In roasted almonds undergoing accelerated shelf life. J. Agric. Food Chem. 65, 2549-2563 (2017).

36. Oliveira, I., Malheiro, R., Meyer, A. S., Pereira, J. A. \& Gonçalves, B. Application of chemometric tools for the comparison of volatile profile from raw and roasted regional and foreign almond cultivars (Prunus dulcis). J. Food Sci. Technol. 56, 3764-3776 (2019). 
37. Yeretzian, C., Jordan, A., Brevard, H. \& Lindinger, W. On-Line Monitoring of Coffee Roasting by Proton-Transfer-Reaction Mass-Spectrometry. In: Flavor Release. 10-112 (American Chemical Society, 2000).

38. Wieland, F. et al. Online monitoring of coffee roasting by proton transfer reaction time-of-flight mass spectrometry (PTR-ToF-MS): towards a real-time process control for a consistent roast profile. Anal. Bioanal. Chem. 402 2531-2543 (2012).

39. Gloess, A. N. et al. Evidence of different flavour formation dynamics by roasting coffee from different origins: on-line analysis with PTR-ToF-MS. Int. J. Mass spectrom. 365-366, 324-337 (2014).

40. Whitfield, F. B. Volatiles from interactions of Maillard reactions and lipids. Crit. Rev. Food Sci. Nutr. 31, 1-58 (1992).

41. Batool, Z. et al. Determination of furan and its derivatives in preserved dried fruits and roasted nuts marketed in China using an optimized HS-SPME GC/ MS method. Eur. Food. Res. Technol. https://doi.org/10.1007/s00217-02003556-2 (2020).

42. Hao, J., Xu, X. L., Jin, F., Regenstein, J. M. \& Wang, F. J. HS-SPME GC-MS characterization of volatiles in processed walnuts and their oxidative stability. J. Food Sci. Technol. 57, 2693-2704 (2020).

43. Gong, Y., Kerrihard, A. L. \& Pegg, R. B. Characterization of the volatile compounds in raw and roasted Georgia pecans by HS-SPME-GC-MS. J. Food Sci. 83, 2753-2760 (2018)

44. Wang, S., Adhikari, K. \& Hung, Y. C. Acceptability and preference drivers of freshly roasted peanuts. J. Food Sci. 82, 174-184 (2017)

45. Kwak, J. et al. Volatile organic compounds released by enzymatic reactions in raw nonpareil almond kernel. Eur. Food Res. Technol. 241, 441-446 (2015).

46. Yang, J. et al. Shelf-life of infrared dry-roasted almonds. Food Chem. 138, 671-678 (2013)

47. Esfahlan, A. J., Jamei, R. \& Esfahlan, R. J. The importance of almond (Prunus amygdalus L.) and its by-products. Food Chem. 120, 349-360 (2010).

48. Mexis, S. F., Badeka, A. V. \& Kontominas, M. G. Quality evaluation of raw ground almond kernels (Prunus dulcis): effect of active and modified atmosphere packaging, container oxygen barrier and storage conditions. Innov. Food Sci. Emerg. Technol. 10, 580-589 (2009).

49. Lee, J., Xiao, L., Zhang, G., Ebeler, S. E. \& Mitchell, A. E. Influence of storage on volatile profiles in roasted almonds (prunus dulcis). J. Agric. Food Chem. 62, 11236-11245 (2014)

50. Valdés, A. et al. Monitoring the oxidative stability and volatiles in blanched, roasted and fried almonds under normal and accelerated storage conditions by DSC, thermogravimetric analysis and ATR-FTIR. Eur. J. Lipid Sci. Technol. 117 1199-1213 (2015).

51. Agila, A. \& Barringer, S. Effect of roasting conditions on color and volatile profile including HMF level in sweet almonds (Prunus dulcis). J. Food Sci. https://doi.org/10.1111/j.1750-3841.2012.02629x (2012).

52. Farneti, B. et al. Development of a novel phenotypic roadmap to improve blueberry quality and storability. Front. Plant Sci. 11, 1-21 (2020).

53. Di Guardo, M. et al. ASSIsT: an automatic SNP scoring tool for in- and outbreeding species. Bioinformatics 31, 3873-3874 (2015).

54. Alioto, T. et al. Transposons played a major role in the diversification between the closely related almond and peach genomes: results from the almond genome sequence. Plant J. 101, 455-472 (2020).

55. Sánchez-Pérez, R. et al. Mutation of a bHLH transcription factor allowed almond domestication. Science 364, 1095-1098 (2019).

56. Verde, I. et al. The Peach v2.0 release: high-resolution linkage mapping and deep resequencing improve chromosome-scale assembly and contiguity. BMC Genomics 18, 225 (2017).

57. Arús, P., Yamamoto, T., Dirlewanger, E. \& Abbott A. G. Synteny in the Rosaceae https://doi.org/10.1002/9780470650349.ch4 (2010).

58. Pritchard, J. K., Stephens, M. \& Donnelly, P. Inference of population structure using multilocus genotype data. Genetics 155, 945-959 (2000).

59. Marchese, A., Bos, R. I., Road, N. \& Malling, E. Short Communication The origin of the self-compatible almond 'Supernova'. Plant Breed. 107, 105-107 (2008).
60. Aranzana, M. J., Abbassi, E. K., Howad, W. \& Arús, P. Genetic variation, population structure and linkage disequilibrium in peach commercial varieties. BMC Genet. https://doi.org/10.1186/1471-2156-11-69 (2010).

61. Cao, K. et al. Genetic diversity, linkage disequilibrium, and association mapping analyses of peach (Prunus persica) landraces in China. Tree Genet. Genomes $\mathbf{8}$, 975-990 (2012)

62. Cao, K. et al. Comparative population genomics reveals the domestication history of the peach, Prunus persica, and human influences on perennial fruit crops. Genome Biol. 15, 1-15 (2014).

63. Li $X$ wei, et al. Peach genetic resources: diversity, population structure and linkage disequilibrium. BMC Genet. https://doi.org/10.1186/1471-2156-14-84 (2013).

64. Liu, S. et al. Linkage disequilibrium in North China and Xingjiang apricot cultivars (Prunus armeniaca L.). In: Acta Horticulturae. 269-284 (International Society for Horticultural Science (ISHS), Leuven, Belgium, 2018).

65. Campoy, J. A. et al. Genetic diversity, linkage disequilibrium, population structure and construction of a core collection of Prunus avium L. landraces and bred cultivars. BMC Plant Biol. 16, 1-15 (2016).

66. Flint-Garcia, S. A., Thornsberry, J. M. \& Buckler, E. S. Structure of linkage disequilibrium in plants. Annu Rev. Plant Biol. 54, 357-374 (2003).

67. Omodei, F. Descrizione e caratterizzazione biometrica di cultivar Siciliane di mandorlo (P. Amygdalus L.) in conservazione 'ex situ'. 2007.

68. R Core Team. R: A Language and Environment for Statistical Computing. (R Foundation for Statistical Computing, Vienna, Austria, 2016).

69. Zhao, S., Guo, Y., Sheng, Q. \& Shyr, Y. Heatmap3: an improved heatmap package with more powerful and convenient features. BMC Bioinforma. 15 , 15-16 (2014).

70. Galili, T. dendextend: An R package for visualizing, adjusting and comparing trees of hierarchical clustering. Bioinformatics 31, 3718-3720 (2015).

71. Wickham, H. ggplot2: Elegant graphics for data analysis. (Springer-Verlag New York, 2016).

72. Rohart, F., Gautier, B., Singh, A. \& Lê Cao, K. A. mixOmics: an R package for 'omics feature selection and multiple data integration. PLoS Comput. Biol. 13, 1-19 (2017).

73. Doyle, J. J. \& Doyle, J. L. A rapid DNA isolation procedure for small quantities of fresh leaf tissue. Phytochem. Bull v.19, 11 (1987).

74. Evanno, G., Regnaut, S. \& Goudet, J. Detecting the number of clusters of individuals using the software STRUCTURE: a simulation study. Mol. Ecol. 14, 2611-2620 (2005).

75. Earl, D. A. \& vonHoldt, B. M. STRUCTURE HARVESTER: a website and program for visualizing STRUCTURE output and implementing the Evanno method. Consen. Genet. Resour. 4, 359-361 (2012).

76. Jakobsson, M. \& Rosenberg, N. A. CLUMPP: a cluster matching and permutation program for dealing with label switching and multimodality in analysis of population structure. Bioinformatics 23, 1801-1806 (2007).

77. Di Guardo, M. et al. Genetic structure analysis and selection of a core collection for carob tree germplasm conservation and management. Tree Genet. Genomes 15, 41 (2019).

78. Endelman, J. B. Ridge Regression and Other Kernels for Genomic Selection with R Package rrBLUP. Plant Genome 4, 250-255 (2011).

79. Benjamini, Y. \& Hochberg, Y. Controlling the false discovery rate: a practical and powerful approach to multiple testing. J. R. Stat. Soc. Ser. B 57, 289-300 (1995).

80. Storey, J., Bass, A., Dabney, A. \& Robinson, D. qvalue: Q-value estimation for false discovery rate control. R package version 2.20.0. http://github.com/ jdstorey/qvalue (2020).

81. Laidò, G. et al. Linkage disequilibrium and genome-wide association mapping in tetraploid wheat (Triticum turgidum L.). PLOS ONE https://doi.org/10.1371/ journal.pone.0095211 (2014).

82. Breseghello, F. \& Sorrells, M. E. Association mapping of kernel size and milling quality in wheat (Triticum aestivum L.) cultivars. Genetics 172, 1165-1177 (2006). 